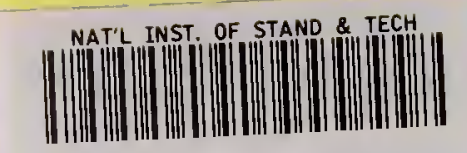

\title{
AN APPROACH TO THE METROLOGICALLY SOUND TRACEABLE ASSESSMENT OF THE CHEMICAL PURITY OF ORGANIC REFERENCE MATERIALS
}

David L. Duewer Reenie M. Parris Edward White V

Willie E. May

Howard Elbaum

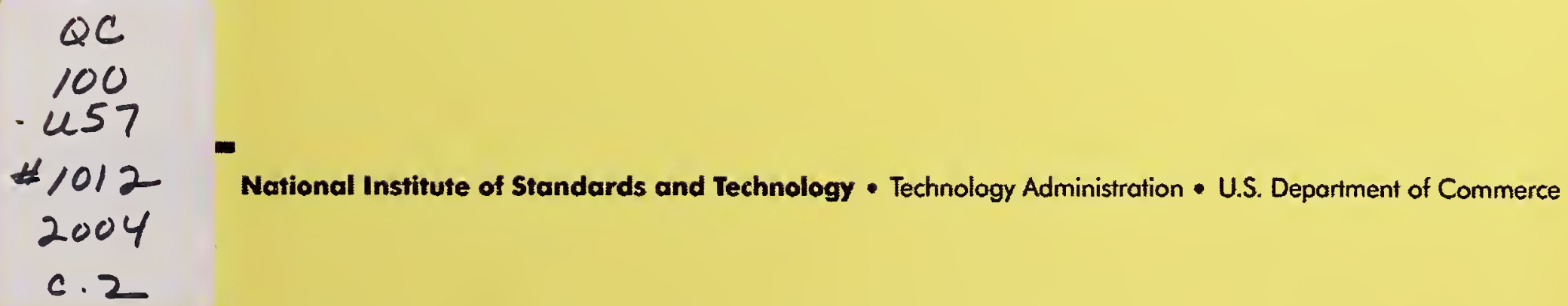





\section{AN APPROACH TO THE METROLOGICALLY SOUND TRACEABLE ASSESSMENT OF THE CHEMICAL PURITY OF ORGANIC REFERENCE MATERIALS}

David L. Duewer, Reenie M. Parris, Edward White V, Willie E. May Analytical Chemistry Division Chemical Science and Technology Laboratory National Institute of Standards and Technology

Gaithersburg, MD 20899

Howard Elbaum $A M S S B-R C B-R Q$ U.S. Army Soldier and Biological Chemical Command Aberdeen Proving Ground, MD 21010

September 2004

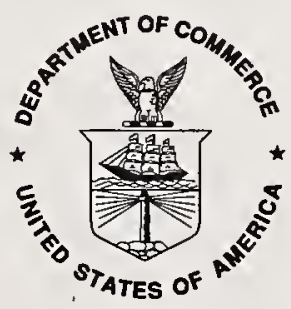

U.S. Department of Commerce Donald L. Evans, Secretary

Technology Administration Phillip J. Bond, Under Secretary for Technology 
Certain commercial entities, equipment, or materials may be identified in this document in order to describe an experimental procedure or concept adequately. Such identification is not intended to imply recommendation or endorsement by the National Institute of Standards and Technology, nor is it intended to imply that the entities, materials, or equipment are necessarily the best available for the purpose.

National Institute of Standards and Technology Special Publication 1012

Natl. Inst. Stand. Technol. Spec. Publ. 1012, 53 pages (September 2004) CODEN: NSPUE2 


\section{ABSTRACT}

This document provides guidelines for the evaluation of the purity of organic chemicals, usually high-purity chemicals, intended for use as reference materials and for the certification of these materials such that assigned values can be considered as metrologically sound and traceable to base units of mass and amount of substance.

The existing methodology for the analysis and certification of high-purity reference materials is presented in sections on metrological concepts and nomenclature, procedures used for preparation and certification of high-purity reference materials including analytical methods, and the appropriate combining of different measurements. The consequences of decisions on required purity, the nature of allowed impurities, traceability to other standards, quantitative characterization, measurement uncertainties, and what information needs to be included in a certificate are considered.

A general approach to the assessment of purity is proposed. The approach includes the determination of what is required for a material to be fit for its intended use, the identification of impurities, the determination of purity by direct or indirect means with a discussion of appropriate analytical techniques, the combining of various analytical results, and the proper presentation of available information. The certificates for a number of materials are evaluated against this approach as case studies.

Four opportunities for research that would enhance the infrastructure available for methods applicable to creating traceable high-purity organic standards are identified. These are: 1) the creation of a limited set of certified materials for quantitative nuclear magnetic resonance, 2) the generation and evaluation of data that would permit estimating uncertainties for measurements based on relative signals from techniques such as gas chromatography and quantitative nuclear magnetic resonance applied to the determination of impurities, 3) the improvement of the prediction of chromatographic detector response factors through the use of quantitative structureproperty relationships, and 4) the development of a sensitive universal mass detector for liquid chromatography with isotope ratio mass spectrometry and chemical reaction interface mass spectrometry as promising candidates.

\section{KEYWORDS}

Chemical metrology, Chemical purity, Reference materials, Traceability 


\section{AN APPROACH TO THE METROLOGICALLY SOUND TRACEABLE ASSESSMENT OF THE CHEMICAL PURITY OF ORGANIC REFERENCE MATERIALS}

\section{TABLE OF CONTENTS}

ABSTRACT iii

KEYWORDS iii

TABLE OF CONTENTS . $\mathbf{v}$

GLOSSARY 1

1. INTRODUCTION. .3

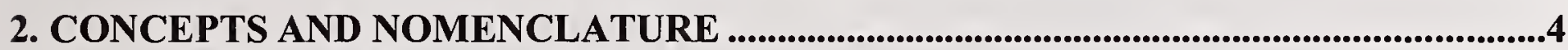

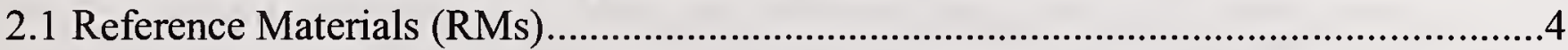

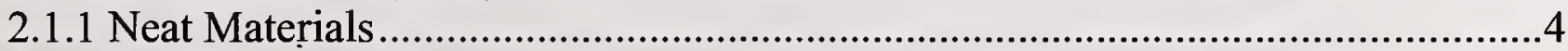

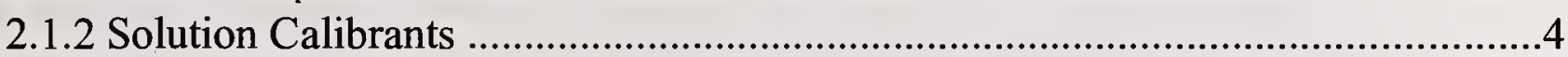

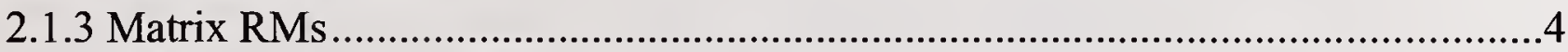

2.2 Certified Reference Materials (CRMs) ………..........................................................

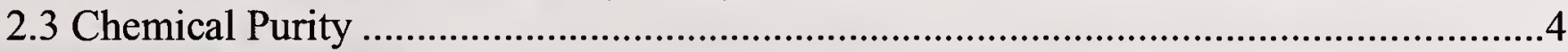

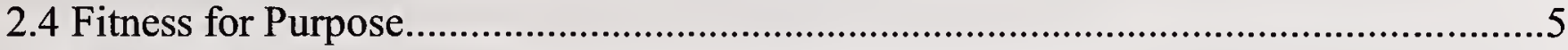

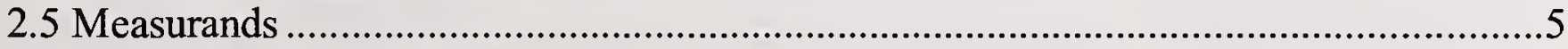

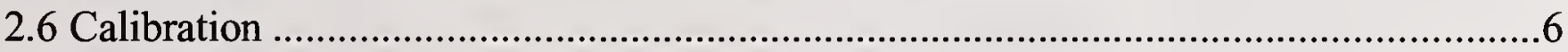

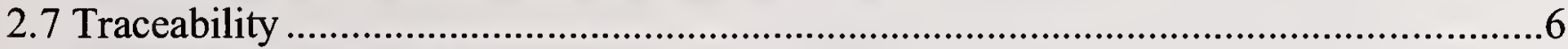

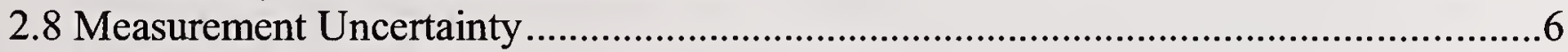

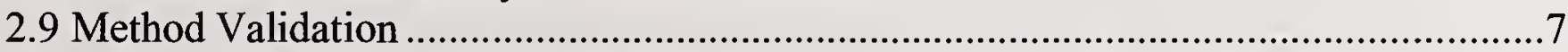

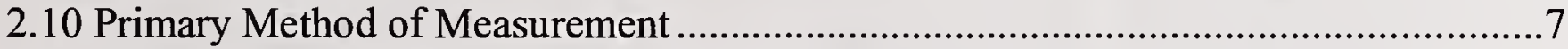

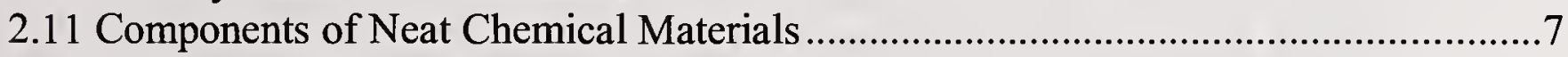

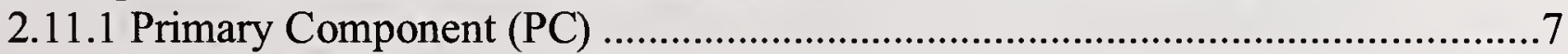

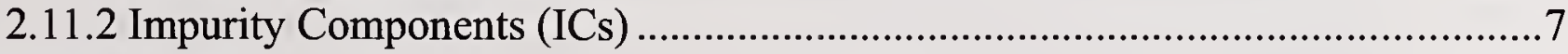

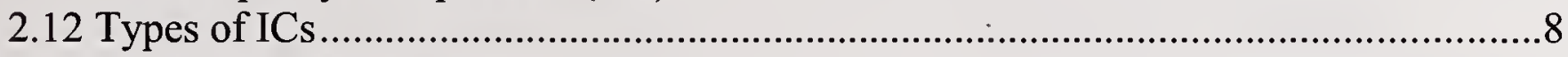

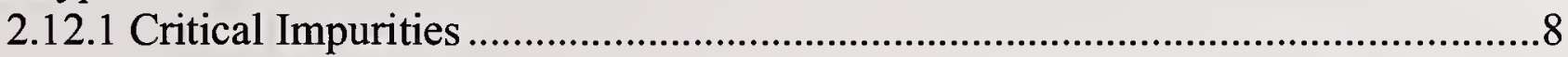

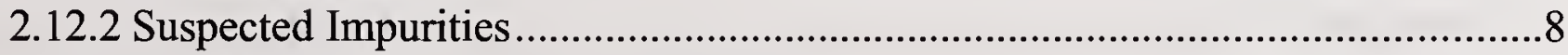

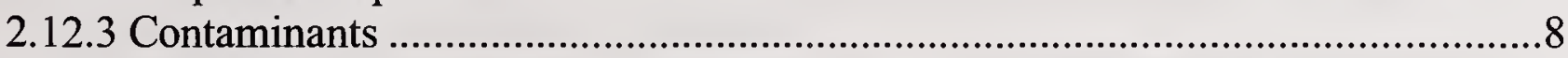

3. BASIC PROCEDURE FOR DEVELOPMENT OF A NEAT-MATERIAL RM ................9

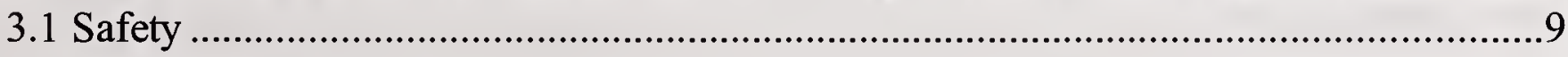

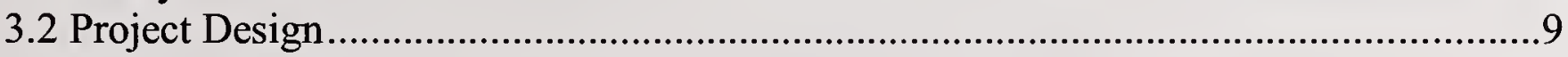

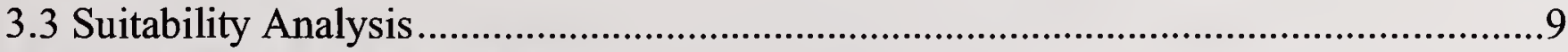

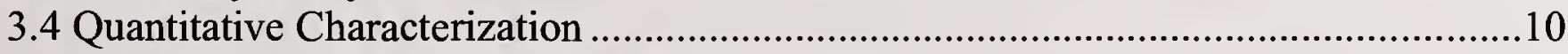

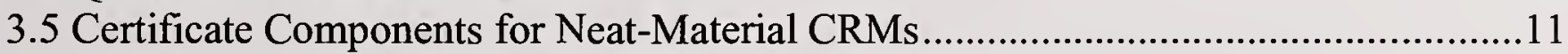


4. METHODS OF CHEMICAL PURITY ANALYSIS ...........................................................12

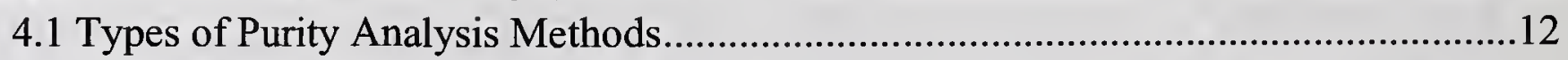

4.2 Direct Determination of the PC Amount ...............................................................13

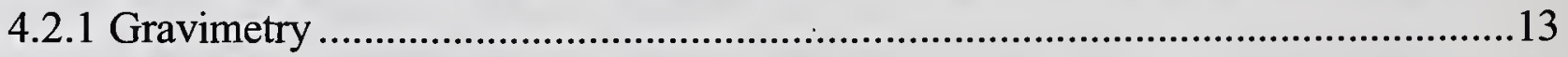

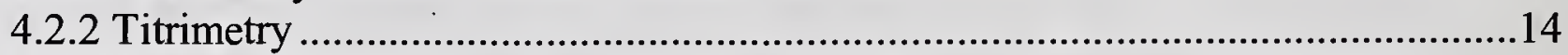

4.2.3 Internal Standard Quantitative Nuclear Magnetic Resonance (IS-qNMR) ........14

4.2.4 Stable Isotope Ratio Mass Spectrometry ............................................................15

4.2.5 Cavity Ring-Down Spectroscopy (CRDS) …..................................................16

4.3 Direct Determination of the PC Mole or Mass Fraction...............................................16

4.3.1 Area Percent Gas Chromatography (\%GC) ……..............................................16

4.3.2 Area Percent Liquid Chromatography (\%LC) ...............................................17

4.3.3 Area Percent Quantitative Nuclear Magnetic Resonance (\%qNMR).................18

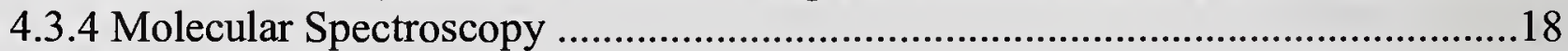

4.3.5 Elemental Analysis ............................................................................... 19

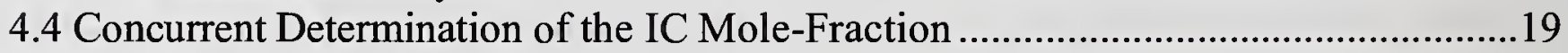

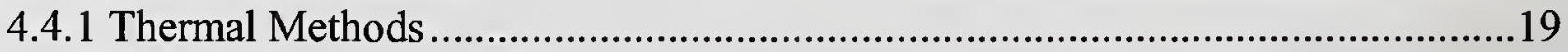

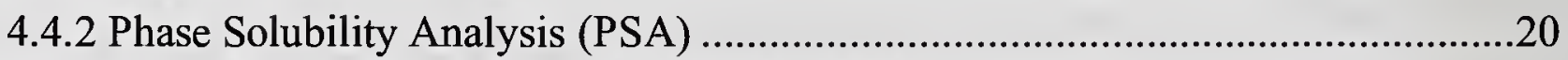

4.5 Consecutive Determination of the IC Mole or Mass Fraction.................................21

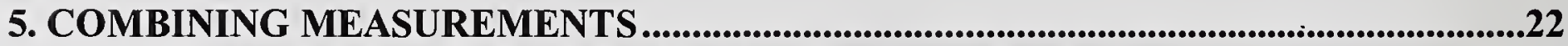

5.1 Measurements of the Same Measurand Can Be Averaged .........................................22

5.2 Measurements of Different Measurands Can Be Summed.........................................23

5.3 Measurements of Not Quite the Same or Completely Different Measurands............24

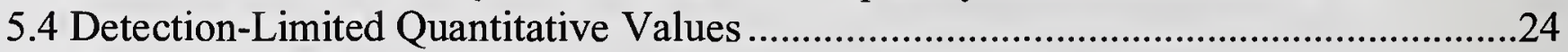

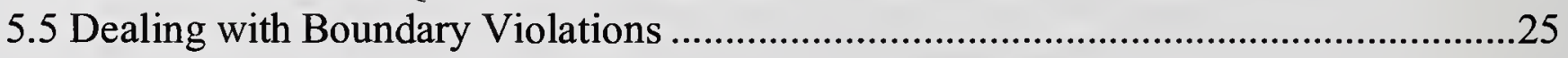

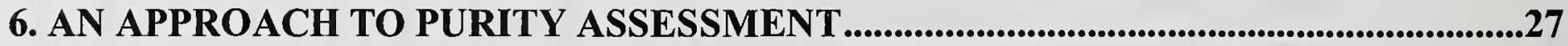

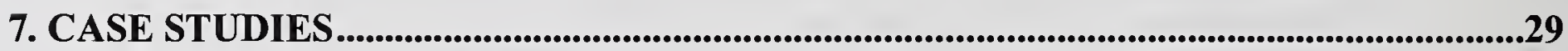

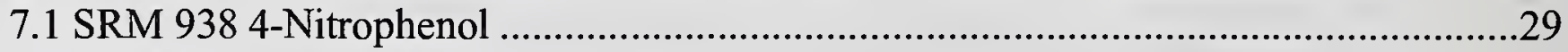

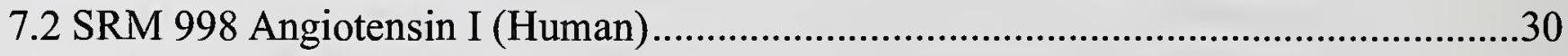

7.3 SRM 910 Sodium Pyruvate ………......................................................................

7.4 LGC1 110 (pp'-Dichlorodiphenyl)dichloroethylene (pp'-DDE) ….............................32

7.5 Arsenobetaine Master Material for BCR-626 ...........................................................33

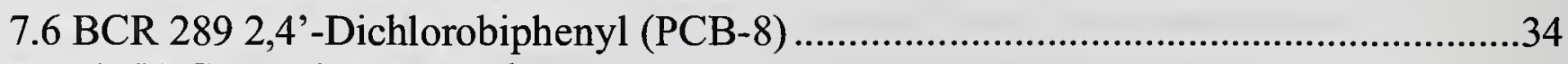

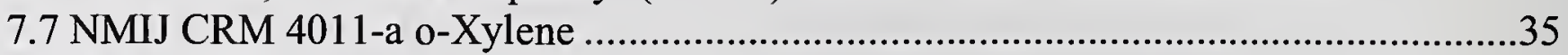

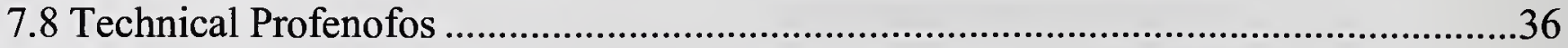

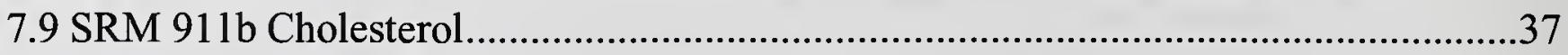

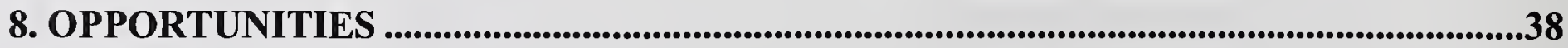

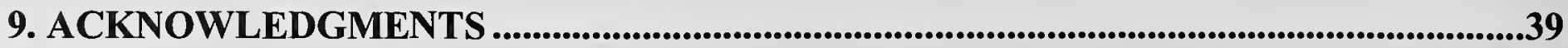

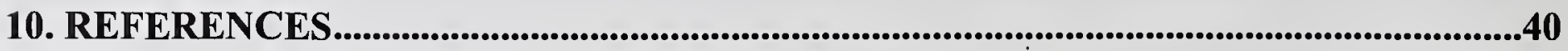




\section{GLOSSARY}

AED

BCR

CRDS

CRIMS

CRM

DSC

ECD

ELSD

FID

Fl

GC

IC

ICP

ID

IRMS

IS

$L B_{95}$ (quantity) Lower Bound of the $95 \%$ confidence interval on the specified quantity

LC

LGC

LoD

NIST

NMIJ

$m_{\mathrm{C}}$

$m_{\mathrm{IC}}$

$M_{\mathrm{IC}}$

$m_{\mathrm{PC}}$

$M_{\mathrm{PC}}$

MS

NAA

$n_{\mathrm{C}}$

$n_{\mathrm{IC}}$

NMR

$n_{\text {ndIC }}$

$n_{\mathrm{PC}}$

OES

PC

PSA

qNMR

QSPR

RM

SI

SRM®

$u$ (quantity)

$U_{95}$ (quantity)

$U B_{95}$ (quantity)Upper Bound to the $95 \%$ confidence interval on the specified quantity

Atomic Emission Detection

Community Bureau of Reference, Brussels, Belgium

Cavity Ring-Down Spectroscopy

Chemical Reaction Interface Mass Spectrometry

Certified Reference Material, an RM with recognized pedigree

Differential Scanning Calorimetry

Electron Capture Detection

Evaporative Light Scattering Detection

Flame Ionization Detection

Fluorescence detection

Gas Chromatography

Impurity Component

Inductively Coupled Plasma ionization

Isotope Dilution

Isotope Ratio Mass Spectrometry

Liquid Chromatography

Laboratory of the Government Chemist, Teddington, England, UK

Limit of Detection

National Institute of Standards and Technology, Gaithersburg, MD, USA

National Metrology Institute of Japan, Tsukuba, Ibaraki, Japan

mass (g) of any component of a material

mass $(\mathrm{g})$ of an IC

relative molar mass (molecular weight, $\mathrm{g} / \mathrm{mol}$ ) of an IC

mass $(\mathrm{g})$ of the PC

relative molar mass (molecular weight, $\mathrm{g} / \mathrm{mol}$ ) of the PC

Mass Spectrometry

Neutron Activation Analysis

amount (mol) of any component of a material

amount (mol) of an IC

Nuclear Magnetic Resonance

maximum likelihood estimate of the amount (mol) of a non-detected IC amount (mol) of the PC

Optical Emission Spectroscopy

Primary Component

Phase Solubility Analysis

quantitative Nuclear Magnetic Resonance

Quantitative Structure-Property Relationship

Reference Material

Système International d'unités

Standard Reference Material, a CRM pedigreed by NIST

standard uncertainty (standard deviation) on the specified quantity

95\% expanded uncertainty on the specified quantity

UB. 
UV/Vis UltraViolet-Visible absorbance detection

$w_{\mathrm{C}}$ mass of substance fraction $(\mathrm{g} / \mathrm{g})$ of any component of the material

$w_{\mathrm{IC}}$

$w_{\mathrm{PC}}$

$x_{\mathrm{C}}$ mass of substance fraction $(\mathrm{g} / \mathrm{g})$ of an IC mass of substance fraction $(\mathrm{g} / \mathrm{g})$ of the PC

$x_{\mathrm{IC}}$

$x_{\mathrm{PC}}$

$\mathrm{Y}$

$Y_{j}$

$\% \mathrm{GC}$

$\% \mathrm{LC}$

amount-of-substance fraction $(\mathrm{mol} / \mathrm{mol})$ of any component of the material amount-of-substance fraction $(\mathrm{mol} / \mathrm{mol})$ of an IC amount-of-substance fraction $(\mathrm{mol} / \mathrm{mol})$ of the PC a specific measurand the $j^{\text {th }}$ expected value of $Y$

$\mathrm{GC}$ analysis based upon the ratio of peak areas

$\% \mathrm{qNMR}$ LC analysis based upon the ratio of peak areas

$\left\{Y_{j}, U_{95}\left(Y_{j}\right)\right\}$ $\sum \mathrm{LoD}$

$\sum m_{\mathrm{IC}}$

$\sum n_{\mathrm{IC}}$

$\sum n_{\text {ndIC }}$

$\sum w_{\mathrm{IC}}$ qNMR analysis based upon the ratio of signal areas

a nominally valid measurement result \{expected value, $95 \%$ uncertainty of $Y$ sum of LOD for all relevant ICs

$\sum x_{\text {IC }}$ total mass $(\mathrm{g})$ of all the ICs total amount (mol) of all the ICs maximum likelihood estimate of the total amount (mol) of all non-detected ICs total mass of substance fraction $(\mathrm{g} / \mathrm{g})$ of all the ICs total amount-of-substance fraction $(\mathrm{mol} / \mathrm{mol})$ of all the ICs 


\section{INTRODUCTION}

This report surveys the analytical techniques that have been used in the assessment of the purity of organic chemicals. It proposes a general approach for purity characterization that fulfills the requirements of, and should be recognized as, sound chemical metrology. When conscientiously applied to the purity-characterization of a reference material (RM), this approach traceably links the certified purity value of the RM to the base Système International d'unités (SI) units of mass (kilogram) and amount of substance (mole) [1]. In combination with other aspects of sound metrology, this linkage enables meaningful comparison of measurements made in different laboratories and at different times.

Establishing the traceability of statements of chemical purity to SI units in principle requires complete knowledge of the composition of the material analyzed. However, complete knowledge of the chemical composition of any real substance is an impractical if not unrealizable goal. Traceability of chemical measurements to the SI is therefore practically realizable through substances appropriately characterized with respect to their chemical structure (qualitative analysis) and composition (quantitative analysis) [2,3]. These substances are usually high-purity (neat) materials.

Despite the central role of neat materials in establishing traceability, there is remarkably little literature guidance on how to evaluate such materials for chemical purity - particularly for structurally complex organic molecules. Formal requirements for assessing measurement quality through method validation, uncertainty evaluation, and traceability demonstration are quite recent, dating from the early 1990s [4]. However, the most complete review of analytical methods useful for evaluating organic purity reflects the analytical capabilities of the late $1960 \mathrm{~s}$ [5].

Section 2 of this document introduces relevant metrological concepts and nomenclature. Section 3 reviews the general RM development process, with an emphasis on how the evaluation of neat materials differs from the evaluation of other chemical RMs. Section 4 presents and evaluates the analytical methods that have been used or proposed for traceable analysis of neatmaterial RMs. Section 5 suggests ways of combining various types of measurement results. Section 6 outlines an approach to purity assessment using analytical information from multiple methods to achieve a metrologically sound traceable assessment of material purity. Section 7 discusses the assessment of several different neat-material RMs done in the past relative to the approach proposed in Section 6. Section 8 presents four opportunities for enhancing the analytical infrastructure for the traceable assessment of chemical purity. 


\section{CONCEPTS AND NOMENCLATURE}

The following concepts and terms are essential or useful for discussing purity assessment within the context of chemical metrology.

\subsection{Reference Materials (RMs)}

An RM is a "material or substance one or more of whose property values are sufficiently homogeneous and well established to be used for the calibration of an apparatus, the assessment of a measurement method, or for assigning values to materials" [6].

There are three basic types of RM used in chemical analysis:

\subsubsection{Neat Materials}

A neat material RM is a nominally single-entity material. These RMs are typically used to prepare solution RMs and value assign matrix RMs.

\subsubsection{Solution Calibrants}

A solution calibrant is an RM prepared as a mixture of a diluent with one or more materials of established chemical composition. These RMs may be intended for use as supplied and/or following further dilution by the user.

\subsubsection{Matrix RMs}

A matrix RM is material of composition similar to that of "real samples" that contains a characterized amount of one or more chemical entities. Matrix RMs are typically homogenized natural materials with the entities of measurement interest present at endogenous levels, augmented endogenous levels, or as added material.

\subsection{Certified Reference Materials (CRMs)}

A CRM is a "reference material, accompanied by a certificate, one or more of whose property values are certified by a procedure which establishes its traceability to an accurate realization of the unit in which the property values are expressed, and for which each certified value is accompanied by an uncertainty at a stated level of confidence" [6].

\subsection{Chemical Purity}

While there is no formal metrological definition of either purity or impurity [7], the relevant common usages are "the quality of being not mixed with anything else" and "the quality or condition of containing some extraneous or foreign admixture, especially of an inferior or baser kind" [8]. More specifically: "A sample is sufficiently pure when its properties which are to be investigated or used are representative of those of the main component within certain proscribed limits of errors. In other words: a sample is sufficiently pure when the amount of each of the impurities which may interfere with the specific purpose for which the sample is required is so low that their combined effect is negligible within the desired limits of accuracy" [9]. 
Thus, chemical purity is defined by the amount of desired material in a sample relative to the total amount of all materials. The first step of purity determination is confirmation of the chemical identity of the material. The degree of chemical purity that is acceptable is contingent upon the needs of the intended use(s) of the material - i.e., its fitness for purpose. Materials are inadequately pure for use as RMs and CRMs when the impurities significantly influence the chemical or physical properties of interest.

\subsection{Fitness for Purpose}

The fitness for purpose of chemical measurements is formally defined as the "degree to which data produced by a measurement process enable a user to make technically and administratively correct decisions for a stated purpose" [10]. A key element in the concept is for the "interested parties to define in advance the acceptable degree of measurement uncertainty and desired degree of identification confidence" [11].

In addition to being the criterion for assessing when any aspect of the measurement effort is adequately complete, fitness-for-purpose considerations are central to the prospective design of a measurement study $[12,13]$. The better defined the purpose, the more realistic the forecast of analytical effort required to achieve fitness. An unrealistic, unclear, or overly broad purpose may result in unnecessary costs, delay, or failure of a measurement study.

\subsection{Measurands}

A measurand is a "particular quantity subject to measurement" [6]. Within the context of organic purity determinations, the critical measurands are the amounts of particular substances in a given sample.

Considerable effort may be required to define adequately the set of discrete chemical entities that constitute a given measurand. Some of the definitional issues include:

- Isotopomers: Molecular entities differing only in isotopic composition. Unnatural isotope abundances can affect the results of measurements performed using a wide variety of analytical techniques. Unexpected isotopic abundances may arise from the use of unusual source materials or be induced by manufacturing processes [14].

- Stereoisomers: Molecular entities having the same number and type of atoms and the same bonds among the atoms but differing in the relative three-dimensional orientation of various substructures. While some types of stereoisomers have essentially identical chemical reactivities, all types of stereoisomers may have vastly different biological activities. Nominally identical compounds prepared synthetically and from biological sources may represent quite different sets of molecular entities.

- Rotamers and tautomers: Entities that interconvert with one another under typical laboratory conditions but that differ in their relative two-dimensional orientation due to restricted rotation or the nature of their bonds. While the discrete compounds may have quite different properties, they often exist as equilibrium mixtures and are difficult to isolate. 
Fitness-for-purpose considerations dictate the descriptive detail required for appropriate specification of amount-of-substance measurands.

\subsection{Calibration}

Calibration is a "set of operations that establish, under specific conditions, the relationship between values of quantities indicated by a measuring instrument or measuring system, or values represented by a material measure or a reference material, and the corresponding values realized by standards" [6]. "It is the calibration process that transfers a reference value, usually an International System (SI) unit, to the artifact or instrument under calibration and hence establishes the 'unbroken chain of comparisons' required for traceability." [15]

\subsection{Traceability}

Traceability is the "property of the result of a measurement or the value of a standard whereby it can be related to stated references, usually national or international standards, through an unbroken chain of comparisons all having stated uncertainties" [6]. "[F]or consistent and useful measurement results, it is important both that a chain of comparisons to agreed reference standards, and the uncertainties associated with these standards, are established." [16] Traceability is thus a way of ensuring that measurements made at different times, by different analysts, or with different methods can be confidently compared.

Given a suitable RM for a particular chemical measurand, a traceable determination of the amount of that measurand in a given test sample is straight forward - if exacting - analytical chemistry. While requiring rigorously validated fit-for-purpose analytical methods and an appropriate evaluation of the total uncertainty of the measurement, calibration to the RM traceably links the measurement to the RM [15]. If the RM is a CRM (which, by definition, is linked to a higher-order metrological standard), then the measurement is linked through the CRM to that higher-order standard [2].

In the absence of an appropriate RM for a particular measurand, establishing traceability requires linking the measurement either to some appropriate chemical comparator or to physical property(ies) or physical principle(s) for which reference standard(s) are available $[17,18]$. While typically requiring greater resources than direct calibration, such linkages are required whenever the measurand of interest is insufficiently stable, when establishing a CRM, and when the measurement uncertainty must be as small as possible.

\subsection{Measurement Uncertainty}

Measurement uncertainty is formally defined as a "parameter, associated with the result of a measurement, that characterizes the dispersion of the values that could reasonably be attributed to the measurand.... It is understood that the result of the measurement is the best estimate of the value of the measurand, and that all components of uncertainty, including those arising from systematic effects, such as components associated with corrections and reference standards, contribute to the dispersion." [19] 


\subsection{Method Validation}

Method validation is formally defined as "confirmation by examination and the provision of objective evidence that the particular requirements for a specific intended use are fulfilled" [20]. The primary purpose of analytical validation is to "clearly define the application area and the total reliability" of the methods [21].

\subsection{Primary Method of Measurement}

A primary method has been described as "a method having the highest metrological qualities, whose operation can be completely described and understood, for which a complete uncertainty statement can be written down in terms of SI units. A primary direct method measures the value of an unknown without reference to a standard of the quantity. A primary ratio method measures the value of a ratio of an unknown to a standard of the same quantity; its operation must be completely described by a measurement equation." [22,23] "First, it must be a method which is specific for a defined substance and second, the values of all parameters, or corrections which depend on other species or the matrix, must be known or calculable with appropriate uncertainty." [17]

More recent commentaries recognize that there are no measurement methods or technologies that operate at a primary level for all applications. Rather, there are a number of general methods that have the potential of meeting the above requirements under specific circumstances for selected measurands. The boundary conditions and scope of applicability for all such methods must always be clearly stated and the method must be validated for the specific use [24].

\subsection{Components of Neat Chemical Materials}

At some level, essentially all materials are mixtures. A neat material can be considered to be a mixture of a primary component, $\mathrm{PC}$, and a set of impurity components, ICs.

\subsubsection{Primary Component (PC)}

The $\mathrm{PC}$ is the chemical entity or aggregate of entities that is of primary interest. For neat material RMs, the PC will be the dominant component of the mixture.

\subsubsection{Impurity Components (ICs)}

An IC is any chemical component of the mixture that is not the PC. There are two major sources for ICs: chemical entities present in a material before purification and those that are introduced by purification efforts and material transfers. ICs in the original material that have physico-chemical properties quite similar to those of the PC may be difficult to remove [9]. ICs introduced into the material may have physico-chemical properties that are quite dissimilar to those of the PC. 


\subsection{Types of ICs}

While ICs can be classified in a number of ways, the following three potentially overlapping categories are useful to following discussions.

\subsubsection{Critical Impurities}

Critical impurities are those whose presence at greater than some defined amount-ofsubstance fraction will render the material unfit for purpose.

\subsubsection{Suspected Impurities}

Suspected impurities are those that are at least plausibly in the material either from its known history (especially preparation, purification, and storage) and its likely modes of decomposition (especially thermal stability and reactivity with oxygen, water, and carbon dioxide).

\subsubsection{Contaminants}

Contaminants are those substances that are not anticipated to be present in the material on the basis of the material's known chemical properties or history. The presence of such ICs suggests imperfect knowledge of the material's history and true properties. 


\section{BASIC PROCEDURE FOR DEVELOPMENT OF A NEAT-MATERIAL RM}

Development of a neat-material RM as a primary standard involves the same tasks as does development of any other chemical RM. There is general agreement on the critical technical and procedural requirements for valid RM certification [2, 3, 25 - 27]. The following sections review the major considerations, with emphasis on those areas where the characterization of neatmaterial RMs may differ from practices applied to other types of chemical RMs.

\subsection{Safety}

This document is not intended in any way to cover or set policy or procedures for environmental-, safety-, or health-related compliance or activities. However, it should be recognized that neat organic materials must be handled with due care, in accordance with all pertinent regulations and common sense. Determining the proper laboratory facilities, personal protective devices, and environmental safeguards required for safe and responsible material handling should be among the first considerations when starting any project.

\subsection{Project Design}

For a given problem, the components of the task must be clearly identified and assessed against the available materials, methods, and resources. The PC and any critical ICs must be explicitly identified and fit-for-purpose minimum (for the PC) or maximum (for ICs) amount-ofsubstance fractions established. Both "as pure as possible" and "zero tolerance" are unachievable within finite resources. Sufficient candidate material must be available to accomplish the necessary certification studies and to provide an adequate number of RM units. If fit-for-purpose candidate materials are not available, there must be sufficient resources available to prepare or otherwise obtain fit-for-purpose material.

The physical and chemical properties of the PC must be compatible with the required minimum shelf life for the standard under achievable storage conditions. Plausible qualitative and quantitative methods of determination of the PC and critical IC measurands are required. If specific methods are not available, there must be sufficient resources available to develop and validate the needed techniques from known general approaches.

\subsection{Suitability Analysis}

Once one or more candidate materials have been identified, it is necessary to evaluate as efficiently as practical their fitness for purpose. The material with the highest amount-ofsubstance fraction for the PC may not be the most fit; it may rather be the material that can be most completely characterized - i.e., the material that can be assigned the smallest uncertainty on the amount of PC or critical IC.

Confirmation of the identity of the PC is a central task of purity determination. All validated techniques appropriate for the determination of the identity of the PC should be considered. 
When the physical properties of the material are well-known and distinctive, evaluation of one or more characteristic property such as melting point may suffice. In the more general case, various specialized forms of high-resolution chromatography, nuclear magnetic resonance (NMR), mass spectrometry (MS), and molecular spectroscopy (ultraviolet, visible, infrared, and Raman) may be required.

The presence/effective absence of critical ICs should be assayed and, if detected, the amount of each material at least semi-quantitatively evaluated. All validated techniques appropriate to this purpose should be considered. Typical techniques for organic ICs include many of the "hyphenated" combinations of separation technologies and spectroscopic detection such as gas chromatography with flame ionization (GC-FID) or MS detection (GC-MS) and liquid chromatography (LC) with MS detection (LC-MS). Typical techniques for inorganic ICs include X-ray fluorescence spectrometry, inductively coupled plasma ionization (ICP) in . combination with optical emission spectroscopy (ICP-OES) or MS (ICP-MS), neutron activation analysis (NAA), ashing, and - for water - mass loss on drying or Karl Fischer titration. Identification and quantitative determination may be accomplishable from the same assays.

Once suitable techniques have been established, a worst-case material heterogeneity survey should be conducted. This study should compare several "as different as possible" sub-samples of the material. This may include different containers, different strata (top, middle, bottom) within a single container, and different particle sizes or crystal morphologies. If the material is appreciably heterogeneous, the material should be purified and/or blended to establish adequate uniformity. A minimum sample size for reliable analysis should be identified. This survey may be accomplishable along with quantitative characterization.

\subsection{Quantitative Characterization}

The quantitative characterization of a neat material does differ from that of other RMs. First, in nearly all cases any measurement of the PC must be accomplished via calibration to a chemical comparator different from the PC or to some physical property or principle. Second, the characterization may require many different types of measurements of different chemical components and/or properties. This requires a fit-for-purpose evaluation of the identity of the PC and the nature and amount of impurities in the material. Since "absence of evidence is not evidence of absence" (Sir Martin J. Rees, UK Astronomer Royal), the realistic goal is to assemble a chemically convincing body of evidence that the evaluation

- would have observed all critical and suspected ICs if they had been present at significant levels relative to the material's intended purpose,

- included a sufficiently broad survey for contaminant ICs,

- included a sufficiently sensitive blunder-check comparison of direct and indirect determinations to catch any major overlooked ICs or errors of analysis, and

- the results of all determinations have been appropriately combined (see Section 5).

As with any RM, the homogeneity of the material must be evaluated. As a general rule, the homogeneity study should evaluate the ICs that are present in largest amount, are expected to be most variable or innately susceptible to environmental contamination (such as water), and/or are 
most critical to fitness for purpose. Comparison of multiple lots or sources of the material can help to identify the most variable ICs.

The lifetime and expected shelf life of the material must also be evaluated. These may be assessed from the material's physical and chemical properties and through accelerated aging experiments. Aged samples should be evaluated for any qualitative and quantitative changes in the ICs present.

\subsection{Certificate Components for Neat-Material CRMs}

Ultimately, an RM's utility is determined by its fitness for purpose for a particular measurement and the degree of confidence the user of the RM has in the assigned value(s). In addition to appropriately stating the amount and uncertainty for the PC and other formal requirements [28], to enable a user to appropriately assess the utility of the CRM for a particular purpose the certificate should also:

- state the identity and amounts of all ICs that were quantitatively determined,

- state the identity and limit of detection for other ICs for which the material was explicitly evaluated,

- identify the analytical techniques used to assess the material, including those used to determine identity and to survey for contaminants,

- describe the assumptions and methods used to assess uncertainties and to combine individual results, and

- explicitly assert the traceability of the certified values. 


\section{METHODS OF CHEMICAL PURITY ANALYSIS}

The following discussions summarize the major strengths and weaknesses of analytical technologies for neat-material purity determination. The discussions are not intended to present complete procedures. Many "analytical details" are quite specific to given technologies; the provided references are intended as introductions to the relevant literature. However, some aspects of the analytical process, particularly sample preparation and the selection of representative blanks and controls, are essential for achieving accurate analytical measurements with nearly any technology. Measurement artifacts can be introduced from incomplete processing or introduction of impurity components of reagents and solvents, from contact with container walls, and from environmental exposure. An introduction to these essential if generic aspects of the analytical process is provided in Kolthoff's Treatise on Analytical Chemistry [29].

\subsection{Types of Purity Analysis Methods}

The amount of PC, $n_{\mathrm{PC}}$, plus the amount of all the ICs, $\sum n_{\mathrm{IC}}$, by definition is equal to the total amount of all substances in a sample. Likewise, the mass of PC, $m_{\mathrm{PC}}$, plus the mass of all the ICs, $\sum m_{\mathrm{IC}}$, is equal to the total mass of all substances in a sample. Given a well-characterized $\mathrm{PC}$, amount of substance and mass of substance are readily converted through the PC's relative molar mass (molecular weight), $M_{\mathrm{PC}}$ :

$$
m_{\mathrm{PC}}=n_{\mathrm{PC}} \times M_{\mathrm{PC}} .
$$

Similarly, the amount of substance and mass of substance of every individual IC are converted through each compound's relative molar mass, $M_{\mathrm{IC}}$ :

$$
m_{\mathrm{IC}}=n_{\mathrm{IC}} \times M_{\mathrm{IC}} .
$$

As long as units are consistent, amounts of substance and mass of substance are meaningfully expressed as fractions of the total sample. The amount-of-substance fraction of a component $\mathrm{C}$ (either the PC or an IC), $x_{\mathrm{C}}$, is defined as

$$
x_{\mathrm{C}}=\frac{n_{\mathrm{C}}}{n_{\mathrm{PC}}+\sum_{i} n_{\mathrm{IC}_{i}}}
$$

and the mass of substance fraction of a component $\mathrm{C}, w_{\mathrm{C}}$, is defined as

$$
w_{\mathrm{C}}=\frac{m_{\mathrm{C}}}{m_{\mathrm{PC}}+\sum_{i} m_{\mathrm{IC}_{i}}} .
$$

The $x_{\mathrm{C}}$ and $w_{\mathrm{C}}$ will in general not be equal unless all components have the same $M_{\mathrm{C}}$; however, they do become closer in value as the $M_{\mathrm{C}}$ become similar and the total amount of impurities becomes small relative to the amount of the PC. 
Note that the amount-of-substance fractions and the mass of substance fractions sum to unity

$$
x_{\mathrm{PC}}+\sum_{i} x_{\mathrm{IC}_{i}}=w_{\mathrm{PC}}+\sum_{i} w_{\mathrm{IC}_{i}}=1
$$

If all of the ICs present in the sample can be accounted for, there are four different approaches to determining the purity of a neat material: direct estimation of $n_{\mathrm{PC}}$, direct estimation of $x_{\mathrm{PC}}$ or $w_{\mathrm{PC}}$, concurrent estimation of $1-\sum x_{\mathrm{IC}}$, and consecutive estimation of $1-\sum x_{\mathrm{IC}}$ or $1-\sum w_{\mathrm{IC}}$.

\subsection{Direct Determination of the PC Amount}

The following technologies estimate $n_{\mathrm{PC}}$ without necessarily quantifying all of the ICs.

\subsubsection{Gravimetry}

Gravimetry is not a single method, but rather a collective term for many quite chemically different techniques that have in common the determination of the $n_{\mathrm{C}}$ of a (highly soluble) measurand as a weighable mass of a (highly insoluble) reaction product $[29,30]$. Classical gravimetry is distinct from and should not be confused with gravimetric preparation (preparing an RM by mixing weighed quantities of two or more well-characterized materials), gravimetric titration (metering a titrant by mass rather than volume), or thermogravimetric analysis (mass loss as a function of temperature).

As they rely on accurate weighing and material transfers, gravimetric techniques for organic compounds require destruction of tens of milligrams to many grams of sample. Relative standard uncertainties of less than $0.1 \%$ relative to the mass of the precipitate can be achieved. The methods can be made valid for any $n_{\mathrm{PC}}$. As the $n_{\mathrm{IC}}$ are not determined but $M_{\mathrm{PC}}$ is known, $w_{\mathrm{PC}}$ is the most valid form of fractional determination.

While the basic gravimetric principle depends upon very specific reactivities, relatively few organic compounds can be gravimetrically determined without first transforming them to another chemical entity. PCs that have specific functional moieties or heteroatoms may be transformable into gravimetrically determinable entities, e.g., quantitative oxidation of a sulfur-containing measurand into inorganic oxides (including $\mathrm{SO}_{3}$ ), dissolution into an appropriate solvent followed by precipitation with excess barium chloride, and gravimetric determination of the resulting barium sulfate. However, such conversions are typically not specific; in the example, all sulfur-containing compounds originally in the material will be summed into the final sulfate measurand.

The results of gravimetric measurements are nominally traceable through the mass calibration of the weighing system and the use of transformations that provide a known, fixed stoichiometry for the insoluble reaction product. However, few chemical transformations are entirely specific or complete. In addition to requiring the identification and at least semiquantification of interferent ICs, corrections for losses in any preliminary chemical transformations, incomplete precipitation, co-precipitation, volatile losses, filtration losses, etc. are required. In their purest forms, gravimetric methods are therefore of little practical utility for the traceable determination of organic purity. However, these corrections and their uncertainties may be amenable to determination using fit-for-purpose instrumental methods [31, 32]. 


\subsubsection{Titrimetry}

There are several chemically different classes of titrimetric methods and a vast number of specific techniques within each class $\left[29,30,33\right.$ - 35]. Coulometric titrations determine $n_{\mathrm{PC}}$ via reaction of the measurand (or some chemical transformation of the $\mathrm{PC}$ ) with metered charge transfer. Gravimetric titrations determine $n_{\mathrm{PC}}$ via reaction with a chemical titrant metered by mass. Volumetric titrations determine $n_{\mathrm{PC}}$ via reaction with a chemical titrant metered by volume.

As they rely on accurate metering of the reactant, titrimetric techniques require milligram to gram quantities of sample. The analyses destroy the sample or render it unfit for further characterization. Relative measurement uncertainties of as low as $0.01 \%$ can be achieved. The methods can be made valid for any $n_{\mathrm{PC}}$. As the $n_{\mathrm{IC}}$ are not determined but $M_{\mathrm{PC}}$ is known, $w_{\mathrm{PC}}$ is the most valid form of fractional determination.

Most organic PCs that contain electrochemically or chemically reactive functional groups can be determined with some type of titration, either directly or after chemical transformation. Titrimetric techniques are typically quite functional-group-specific but not compound-specific; any IC that contains a moiety of similar reactivity to that of the PC will consume titrant.

The results of titrimetric measurements are nominally traceable through the calibration of the metering system and, for volumetric and gravimetric methods, through the calibration (standardization) of the titrant. As with gravimetry, these measurements may require correction for non-ideal chemical transformations as well as for interferent ICs. Such corrections and their uncertainties may be amenable to determination using fit-for-purpose instrumental methods [30].

\subsubsection{Internal Standard Quantitative Nuclear Magnetic Resonance (IS-qNMR)}

When immersed in a strong magnetic field a number of atomic nuclei interact with radiofrequency electromagnetic radiation in a manner that, to an excellent first approximation, does not depend upon the chemical composition or bonding of the molecule that contains the nuclei. The fundamental sameness of the transition frequency of each nuclear type is modified by second- and third-order atomic interactions related to their bonding and three-dimensional orientation. These small differences become better resolved and the transition intensity more readily quantified as the magnetic field strength is increased.

The chemical structure-related differences in the transition frequencies make NMR one of the central tools for molecular structure elucidation. Conversely, the almost-but-not-exactlyidentical transition frequencies enable comparison of the number of same-type nuclei in a given chemical environment with the number in another chemical environment. While most commonly used to estimate the relative numbers of nuclei in different chemical environments within one type of molecule, inclusion of an appropriate internal standard (IS) within the sample enables determination of the relative numbers of different types of molecules in the sample [36 - 41]. The internal standard can be of quite different structure from the measurand as long as it contains one or more nuclei of the relevant atomic type. The IS can be mixed with the measurand or, to prevent reaction between the measurand and the IS, it can be isolated inside a coaxial insert within the sample holder [41 - 43]. While potentially complicating the analysis, a variety of solvents are available for use with solid or viscous samples [42]. 
IS-qNMR methods are valid for any $w_{P C}$ level. As the $n_{1 \mathrm{C}}$ are not determined but $M_{\mathrm{PC}}$ is known, $w_{\mathrm{PC}}$ is the most valid form of fractional determination.

The Table below lists commonly exploited NMR-active nuclei, their typical natural abundances and relative sensitivities, and an indication of how frequently they have been used in qNMR studies. Most organic materials contain one or more of these nuclei. Typically a sample size of a few to several hundred milligrams is required. Isolation of the IS from the measurand allows recovery of the measurand for other studies. Relative measurement uncertainties of $0.5 \%$ and less are achievable [44].

NMR-Active Nuclei Used in qNMR Studies [45]

\begin{tabular}{ccccc} 
Nucleus & $\begin{array}{c}\text { \% Natural } \\
\text { Abundance }\end{array}$ & $\begin{array}{c}\text { Sensitivity Relative to } \\
\text { Same Number of } \\
\text { Atoms }\end{array}$ & $\begin{array}{c}\text { Abundance } \times \\
\text { Relative } \\
\text { Sensitivity }\end{array}$ & $\begin{array}{c}\text { Number } \\
\text { qNMR } \\
\text { Publications }\end{array}$ \\
\cline { 1 - 5 }${ }^{1} \mathrm{H}$ & 99.98 & 1 & 1 & Many \\
${ }^{19} \mathrm{~F}$ & 100 & 0.833 & 0.8 & Few \\
${ }^{31} \mathrm{P}$ & 100 & 0.066 & 0.07 & Several \\
${ }^{17} \mathrm{O}$ & 0.037 & 0.029 & 0.001 & Several \\
${ }^{29} \mathrm{Si}$ & 4.70 & 0.008 & 0.0003 & Few \\
${ }^{13} \mathrm{C}$ & 1.11 & 0.016 & 0.0002 & Many \\
${ }^{15} \mathrm{~N}$ & 0.37 & 0.001 & 0.000004 & None found
\end{tabular}

The results of single-pulse IS-qNMR measurements are traceable to the amount of substance of the internal standard used. The quantitative utility of multiple-pulse methods using decoupling or cross polarization techniques of any sort must be carefully evaluated on a case-bycase basis. Since the actual comparison is the number of nuclei in one chemical environment relative to the number in a second environment, only one isolated signal each from the IS and the material of interest is required for comparison. However, all of the relevant NMR transitions for both materials should be characterized prior to selecting the particular transitions to be used in the comparison.

\subsubsection{Stable Isotope Ratio Mass Spectrometry}

There are a number of MS techniques that can be used use to characterize quantitatively components in a mixture, all using the ratio of signals from two or more stable isotopes of given atomic types. Isotope dilution MS (IDMS) techniques exploit the change in ratios as a function of the addition of known quantities of an isotopically enriched version of the same measurand. While often sufficient in itself for the analysis of calibration solution and matrix RMs [46 - 48], one-step IDMS has limited applicability for the determination of $n_{\mathrm{PC}}$ for neat organics since it requires detailed characterization of the labeled material. Multiple-step IDMS may enable "analyses for which reference materials with certified isotope ratios are not available" [49]. Relative uncertainties of a few $0.01 \%$ to $0.1 \%$ are achievable.

Relative uncertainties of a few $0.1 \%$ can also be achieved by measuring the ratios of reaction products using isotope ratio MS (IRMS) or chemical reaction interface MS (CRIMS) where the 
molecules of interest are converted online to small polyatomic molecules such as $\mathrm{CO}_{2}, \mathrm{NO}$, and $\mathrm{SF}_{6}[50,51]$. Used in combination with chromatographic separations, these methods can be calibrated with suitably labeled comparators that are structurally different from the desired measurand.

Results of measurements made using the isotope ratio MS techniques are traceable to or through the isotopically enriched comparator and, most importantly, to the complete experimental realization of the assumptions of the relevant complete measurement equation.

\subsubsection{Cavity Ring-Down Spectroscopy (CRDS)}

CRDS is a relatively new optical spectroscopy that exploits the time-domain decay of light trapped within an extraordinarily reflective resonance cavity to determine the product of the number density of a specific gaseous absorber and its absorption cross-section. Once the crosssection is known, $n_{\mathrm{PC}}$ can be deduced from measurement without direct use of a chemical comparator.

While CRDS has been represented as a "primary method" for the analysis of trace gases [52, 53], the requirement for accurately determined absorption cross-section suggests that it has very limited applicability for the analysis of neat materials. That is, materials suitable for adequately determining the cross-section would be adequate for the calibration of other analysis techniques. As currently developed, CRDS is suitable only for the determination of molecular entities that have narrow absorption features for which single-mode laser sources are available that can be tuned completely across the feature.

Results of CRDS measurements are traceable to the RM used to define the absorption crosssection.

\subsection{Direct Determination of the PC Mole or Mass Fraction}

If all components of a mixture could be completely resolved as a function of time (for separation systems), frequency (for optical spectroscopic systems), mass (for mass spectroscopic systems), or other physical principle and if each component could be detected with equal molar or molar-mass sensitivity, then the ratio of the signal due to the PC relative to the sum of the signals from all components would directly estimate $x_{\mathrm{PC}}$ or $w_{\mathrm{PC}}$. The following technologies approximate this direct estimation without necessarily quantifying all of the ICs.

\subsubsection{Area Percent Gas Chromatography (\%GC)}

A well-designed and correctly executed determination using capillary column GC can provide very complete separation of mixture components. IRMS, CRIMS, and atomic emission detection (AED) quantitatively convert molecules to their constituent atoms or to a set of characteristic small polyatomic molecules and thus have the potential to be highly sensitive universal molar-mass detectors $[50,51,54]$. These techniques require specialized equipment and expertise; their quantitative performance characteristics have not yet been thoroughly characterized. Of the commonly used detection principles, flame ionization detection (FID) comes closest to providing an adequately sensitive response that is proportional to the relative molar mass [55]. 
FID is quite sensitive for any volatile or semi-volatile molecule that contains $\mathrm{C}-\mathrm{H}$ linkages, has a very large linear dynamic range, and is proportional - to a good first approximation - to the carbon content of the molecule. When all ICs are known to be very similar to the PC (i.e., isomers and close homologues), GC-FID directly estimates $w_{\mathrm{PC}}[56,57]$. Given a suitably volatile material, capillary column GC analysis of a neat material requires very little sample. Relative integration uncertainties of $0.5 \%$ can be achieved. Since the GC-FID (1) is insensitive to water and non-volatile compounds and (2) provides a response to volatile compounds that is only approximately constant even for isomers and close homologues, this approach is never completely valid. Ongoing research into chemical class-specific response factors, linearity of response factors, and associated detection uncertainties may enhance the utility of \%GC-FID for the assessment of neat-materials [58].

Results of \%GC $w_{\mathrm{PC}}$ measurements are traceable through the validity of the assumptions:

- the $w_{\mathrm{PC}}$ is very high,

- the chromatographic separation achieves complete separation of all ICs from the PC,

- all of the ICs elute and are detected, and

- the detector response factors are very similar for the PC and all ICs.

Use of capillary columns, temperature programming, and several different column stationary phases can help validate the suitability of the separation. GC-electron capture detection (GCECD) provides excellent sensitivity for halogenated compounds. GC-MS with library search capability can help confirm the similarity of ICs. GC-IRMS and GC-CRIMS may enable calculation of GC-FID response factors even if molecular structures cannot be uniquely identified (see Section 4.5).

\subsubsection{Area Percent Liquid Chromatography (\%LC)}

While LC systems typically provide somewhat lower resolution than can be achieved with capillary GC, they enable use of a much greater diversity of stationary and mobile phases. Except for very volatile materials, high-performance LC systems may thus enable more complete separation of components than can be achieved with GC alone. Typical LC separations are performed at lower temperatures than $\mathrm{GC}$ and are thus less prone to thermal degradation of the sample material. However, no LC detection system is available that is suitably sensitive and that responds about equally to all organic material.

The widely used ultraviolet-visible absorbance (UV/Vis) and fluorescence (Fl) detectors provide excellent sensitivity for many organic moieties; however, many compounds do not contain UV/Vis- or Fl-active substructures. Further, LC-UV/Vis and LC-Fl response intensities are sensitive to small changes in molecular structure; the common analytical practice of using single-wavelength UV/Vis detection is inappropriate for characterization of unidentified ICs.

Evaporative light scattering detection (ELSD) has been claimed as a step towards a universal LC detection principle [59]. An ELSD detector consists of a nebulizer to convert the eluent to an aerosol, a drift tube to vaporize the liquid-phase solvent, and a light-scattering cell where the detection of any particles takes place. However, LC-ELSD is much less sensitive than GC-FID and has a smaller linear dynamic range. The scattering signal is a complex and not fully understood function of the particle size and how each compound absorbs, refracts, reflects, 
Rayleigh scatters, and Mie scatters the incident light [59]. The net response factors do not yet appear to be quantitatively predictable. At present, LC-ELSD appears to be most useful for semi-quantitative confirmation studies.

While unproven for purity determination, LC-IRMS and LC-CRIMS have the potential for estimating the relative mass as well as at least partial molecular formulas for all ICs [50,60].

\subsubsection{Area Percent Quantitative Nuclear Magnetic Resonance (\%qNMR)}

$\% q$ NMR compares the integrated area for all of the NMR nuclear transitions for a given atomic type that are attributable to the PC to the sum of the area of all observed transitions of the material. If at least one but not all of the PC transitions can be resolved from those of the ICs, the area ratio can be established by suitably correcting the measured area by the ratio of total nuclei to measured nuclei [42].

Unlike the separation-based GC-FID and LC-ELSD methods, all ICs that contain the relevant isotope will contribute to the total integral. To a good first approximation, each relevant atom in the material contributes equally to the total signal intensity. When the relevant isotope is present in very different chemical environments, the relative response factor for each transition frequency is a calculable function of the difference between the transition and the fundamental excitation frequency [39]. If the ICs can be identified, the appropriate atom-to-molecule ratios can be determined and corrected for (see Section 4.5). Relative integration uncertainties of $0.5 \%$ can be achieved, although ICs present at $x_{\mathrm{PC}}$ of less than $\approx 0.1 \%$ may not be fully accounted for.

Comparison of results for different atomic types, particularly ${ }^{1} \mathrm{H}$ - and ${ }^{13} \mathrm{C}-\mathrm{NMR}$, can assist validation. The results of $\%$ qNMR $x_{\mathrm{PC}}$ measurements are traceable through the validity of the assumptions:

- the $x_{\mathrm{PC}}$ is very high,

- all signals have been completely integrated, and

- the atom-to-molecule ratios have been correctly determined.

\subsubsection{Molecular Spectroscopy}

If the molecular spectrum (typically in the ultraviolet, visible, infrared, or near infrared spectral regions) of a material is known then $x_{\mathrm{PC}}$ may be determinable by comparison of an observed spectrum to the known spectrum. If the spectrum of the PC contains one well-defined unique feature then $x_{\mathrm{PC}}$ can be calculated as the ratio between the observed and the known signal for the feature. This is known as zeroth-order calibration; it is appropriate only when none of the potential ICs have any spectral signal in the region of interest. When no single feature provides adequately selective information, a variety of multivariate mathematical techniques may enable estimation of $x_{\mathrm{PC}}$ via comparison of multiple features. This is known as first-order calibration; it is appropriate when the spectra of all the potential ICs are known. When two or more molecular spectroscopies are used to simultaneously generate a matrix of information, as in molecular excitation/emission spectrofluorometery, then recent developments in multivariate chemometrics may enable estimation of $x_{\mathrm{PC}}$ in the presence of unknown ICs. This is known as second-order calibration or $\mathrm{N}$-way analysis [61 - 63]. 
Since zeroth- and first-order calibrations require the absence of, or at least knowledge of the spectral characteristics of, all interferences, they cannot be used to directly estimate $x_{\mathrm{PC}}-$ although they may be useful for consecutive determinations as described in Section 4.5. Only second-order calibration systems are in principle appropriate for direct estimation of PC purity. The precision that can be achieved is a predictable function of the spectral characteristics of the PC, the particular spectroscopic system, and the mathematics used [64]. Relative uncertainties of a few $0.1 \%$ or less have been achieved. Second-order spectroscopic calibrations are traceable to the RM used to define the reference spectrum.

\subsubsection{Elemental Analysis}

There are many techniques that can be used to assay the mole fraction elemental composition of most organic materials [65]. Relative uncertainties of a few $0.1 \%$ have been claimed for the determination of C, H, N, O, P, S, As, Cl, Br, I, and Se. Such assay is very useful as a blunder check - deviation of the observed composition from that expected strongly suggests the presence of contaminant ICs of quite different composition from the PC. However, elemental assay is of no utility for revealing the presence of ICs having the same or very similar molecular formula as the PC.

\subsection{Concurrent Determination of the IC Mole-Fraction}

The following colligative technologies have been claimed to estimate $1-\sum x_{\text {IC }}$ without identifying any of the ICs.

\subsubsection{Thermal Methods}

Given a material that is crystalline at an experimentally accessible temperature, there are numerous methods that directly estimate $\sum x_{\mathrm{IC}}$ by measuring various phase-change related phenomena as a function of temperature [66 - 69]. The more commonly used of these methods are: freezing- and melting-point depression, differential scanning calorimetry (DSC), and adiabatic calorimetry. These methods determine the degree of deviation from the behavior expected of an ideal $100 \%$ pure sample of the material caused by the presence of impurities that are more soluble in the material's liquid phase than in its solid phase. They are applicable when the "pure" material is stable at its melting point and all impurities are soluble in the liquid phase.

Freezing-point and melting-point techniques monitor the fraction of material transformed from one phase to the other as a function of temperature. DSC techniques monitor the relationship between the relative heat flow and the temperature of a sample in comparison to an inert reference. Adiabatic calorimetric methods monitor the absolute heat capacity of a material as a function of sample temperature. Other related techniques monitor volume and dielectric changes in a material as it melts or freezes. These methods generally require $0.001 \mathrm{~g}$ to $0.1 \mathrm{~g}$ amounts of sample. The quantitative physical relationships upon which these techniques are based become more rigorously applicable as $\sum \mathrm{x}_{\mathrm{IC}}$ goes to zero. Precisions of a few $0.1 \%$ or better can be achieved at $\mathrm{X}_{\mathrm{PC}}$ of $\approx 0.99$ or greater. All of the methods become progressively less accurate with declining $\mathrm{X}_{\mathrm{PC}}$; the limit of quantitative utility is $\approx 0.95$. 
The results for all of the thermal methods assert traceability through the validity of their assumptions:

- the $x_{\mathrm{PC}}$ is very high,

- the experiments realized the required thermodynamic conditions,

- there are no insoluble ICs, and

- there are no ICs that form solid solutions.

The differential techniques also require one or more calibrator RMs. DSC has been claimed as a "primary method" for purity [17]; however, in practice DSC often involves empirical corrections that, while of great practical utility, decrease the method's claim to produce traceable results [70]. Of all the current methods, adiabatic calorimetry can best experimentally satisfy its theory's assumptions; it is also the most time and resource intensive [66].

Unfortunately, all colligative techniques are insensitive to ICs that form solid solutions with the PC; i.e., entities that are soluble in both the solid and liquid phases of the PC. Unless the ICs are identified and determined not to be likely to co-crystallize with the PC, the relationship between the character of the phase transition and absolute material purity cannot be adequately established.

\subsubsection{Phase Solubility Analysis (PSA)}

Given a material that is sparingly soluble in some well-defined solvent system and that is crystalline at a temperature that can be maintained quite constant for several weeks, PSA can be used to both estimate $\sum x_{I C}$ and to concentrate the individual ICs for further characterization. PSA monitors the composition of the liquid phase in a series of sealed vials containing increasing amounts of material in fixed amounts of solvent, kept at constant temperature and pressure, as a function of system composition (the amount of the material per unit mass of the material plus the solvent) $[71,72]$.

While it is non-destructive, PSA requires large amounts $(1 \mathrm{~g}$ to $100 \mathrm{~g})$ of sample and several weeks of elapsed time to perform. Due to its dependence on the use of discrete systems, the upper limit to the $x_{P C}$ that can be resolved is $\approx 0.999$. ICs that are more soluble than the PC in the chosen solvent tend to be concentrated into the solvent and thus may be more easily identified in these solutions than they were in the original material [73]. The solvent systems must not chemically react with the PC or any of the ICs.

Like the thermal colligative methods, the results of PSA measurements are traceable through the validity of its assumptions. When care is used to ensure that equilibrium conditions are obtained for all of the discrete systems, the necessary conditions can rather simply be realized for many materials. However, again like the thermal colligative methods, PSA is insensitive to ICs that form solid solutions. Use of two or more solvent systems can help reveal such problems [74]. 


\subsection{Consecutive Determination of the IC Mole or Mass Fraction}

Any and all validated analytical methods may prove useful for the identification and quantitation of ICs. Some composite ICs such as "insoluble particulate matter," "volatiles," or "ash" may be determinable using gravimetric measurements. More generally, specific ICs need to be individually evaluated using appropriate instrumental methods. However, it is unlikely that suitable RMs for all (or most - or any) of the ICs will be available for calibrating any of the methods. How then can these methods be useful in a purity analysis of a neat material?

The "trick" is that the ICs are (rather, should be) present in the material in amounts several orders of magnitude less than the PC. Large relative uncertainties in the determination of small amounts of material may give adequately small absolute uncertainties [31,34].

Assume that some \%area method, such as \%GC-FID, can be expected to give results having a relative standard uncertainty of $5 \%$. If $w_{\mathrm{PC}}=0.99$, the $95 \%$ expanded uncertainty on $w_{\mathrm{PC}}$, $U_{95}\left(w_{\mathrm{PC}}\right)$, is equal to $\pm 2 \times 0.99 \times 0.05= \pm 0.1$; this is unlikely to be adequate. However, if used to analyze an equally "high-purity" calibrant material for some IC that is present at $w_{\mathrm{IC}}=0.01$ then the $U_{95}\left(w_{\text {IC }}\right)$ is equal to $\pm 2 \times 0.01 \times 0.05= \pm 0.001$. In general, the lower the amount of the IC present in the candidate RM (relative to the amount of the most abundant IC detected), the less critical the expected uncertainty in the analysis of the IC.

Thus, given a sufficiently pure candidate RM so that the IC amounts are small, commercial materials can probably be analyzed sufficiently well for use as fit-for-purpose IC calibrants. It is likely that materials that are adequate to establish confidently the identity of the ICs will also be adequate for their calibration.

When an IC has been identified but no commercial material is available within the time and resource constraints of the project, it may be possible to calculate an appropriate response factor for some methods. There are a number of software systems that enable the calculation of NMR spectra from first principles. Several different quantitative structure-property relationship (QSPR) studies have predicted GC response factors for FID, thermal conductivity, and photoionization detection systems to within about $5 \%$ [75 - 77].

The result of a consecutive $1-\sum x_{\text {IC }}$ or $1-\sum w_{\text {IC }}$ determination is traceable through the validity of the assumptions:

- all ICs (or chemical class groups of ICs) have been recognized,

- all ICs have been appropriately quantified, and

- the results of the multiple analyses have been appropriately combined. 


\section{COMBINING MEASUREMENTS}

When no single measurement proves fitness for purpose, chemical purity must be estimated by assembling information from different sources. Combining disparate data into a metrologically acceptable and chemically satisfying conclusion is an exercise in chemical deduction. It is essential that appropriate mathematics be employed, but determining what information can be combined is primarily a scientific - not a statistical - challenge, task, and responsibility.

\subsection{Measurements of the Same Measurand Can Be Averaged}

When the identities of all the detected ICs are known and the amounts of each have been individually determined, it is relatively simple to:

- identify with fit-for-purpose confidence any replicate measurements of the same quantity,

- transform, if necessary, such replicate measures to have consistent units, and

- composite replicates to yield a single quantitative estimate - an expected value and an associated $95 \%$ confidence interval about the expected value.

Given $N$ nominally valid estimates from different analytical methods for measurand Y, how should they be combined to provide the most meaningful estimate of the true value of Y?

Let $\left\{Y_{j}, U_{95}\left(Y_{j}\right)\right\}$ represent the set of $N$ measurements, where $Y_{j}$ is the best estimate of the value from method $j$ and $U_{95}\left(Y_{j}\right)$ is its fully evaluated $95 \%$ expanded uncertainty [19]. When the $Y_{j}$ s substantially coincide (i.e., when the results from different methods agree within their assigned uncertainties) and the $U_{95}\left(Y_{j}\right)$ are approximately equal, an adequate estimate is provided by the simple mean of the methods

$$
\bar{Y}=\sum_{j=1}^{N} Y_{j} / N
$$

and the approximate expanded standard uncertainty of the mean (combining the among-method and the average within-method variances)

$$
U_{95}(\bar{Y})=2 \times \sqrt{\frac{\left(\sum_{j=1}^{N}\left(Y_{j}-\bar{Y}\right)^{2} / N-1\right)+\left(\sum_{j=1}^{N}\left(\frac{U_{95}\left(Y_{j}\right)}{2}\right)^{2} / N\right)}{N}} .
$$

The use of the value 2 as the coverage factor to convert combined standard uncertainties of fully evaluated measurements into approximate $95 \%$ coverage expanded uncertainties is inexact but quite conventional and accepted metrological practice [19]. If the $N$ measurements must be regarded as random draws from a population of possible measurements (e.g., results from an interlaboratory study), a Student's $t$ coverage factor for $N-1$ degrees of freedom may be more appropriate. 
When the $Y_{j}$ s substantially coincide but the $U_{95}\left(Y_{j}\right)$ are very different, an inverse totaluncertainty weighted mean may be a better estimate than the simple mean $[78,79]$. (Total uncertainty includes all among-method as well as within-method uncertainty components.) However, qualitative and semi-quantitative confirmatory results are valuable primarily to demonstrate the lack of bias of the more quantitative measurements. While all available information needs to be included in the evaluation, not every number must be included in the estimate of the expected value.

When the $Y_{j}$ s do not substantially coincide (i.e., when the results from different methods are substantially biased relative to their assigned uncertainties and these biases actually impact fitness for purpose), there is no universally accepted statistical answer. The approximate Bayesian method detailed in Levenson et al. provides reasonable values when some independent knowledge of the method biases is available [80]. However, chemically significant biases among methods that are putatively equal cast strong doubt on the validity of all results. The most rigorous procedure is to determine the root cause(s) of the bias and either correct the results or establish which - if any - of the results are trustworthy.

When resources are insufficient to define fully and account for observed among-method differences, expert opinion may be used to identify the most reasonable composite value and its associated $95 \%$ confidence interval. While use of subjectively evaluated uncertainty components is metrologically valid [19], it degrades confidence in the resulting values and thus in the traceability of the results.

\subsection{Measurements of Different Measurands Can Be Summed}

When the identity of $N$ different ICs is known, the identities are all different, and the amounts of each have been individually assayed, it is simple to:

- transform, if necessary, all measures to consistent units, and

- combine the individual estimates into a single quantitative estimate.

Letting $\left\{Y, U_{95}(Y)\right\}$ now represent the estimated values for different components, the expected value for the combination of all the identified components is just the sum

$$
Y_{\text {Total }}=\sum_{j=1}^{N} Y_{j}
$$

with an approximate $95 \%$ expanded uncertainty of

$$
U_{95}\left(Y_{\text {Total }}\right)=2 \times \sqrt{\sum_{j=1}^{N}\left(\frac{U_{95}\left(Y_{j}\right)}{2}\right)^{2}}=\sqrt{\sum_{j=1}^{N} U_{95}^{2}\left(Y_{j}\right)} .
$$




\subsection{Measurements of Not Quite the Same or Completely Different Measurands...}

Combining collective measurements, such as the sum of $w_{\text {IC }}$ from all unidentified GC-FID and LC-ELSD peaks, is less simple. When the different estimates can be assumed to include the same collections of ICs, they can be averaged as in Section 5.1. When the different collections can be assumed to be quite different, the estimates should be summed as in Section 5.2. When the collections can be assumed to include some - but not all - of the same ICs, the combined estimate should be intermediate between these best- and worst-case possibilities. When the extent of overlap is difficult to establish, then the worst case should be assumed. If the difference between the best- and worst-case estimates is significant relative to fitness for purpose, then the ICs should be further characterized.

Combining amount-of-substance fraction, $x_{\mathrm{C}}$, and mass fraction, $w_{\mathrm{C}}$, estimates is also not simple when some of the $M_{\mathrm{IC}}$ are unknown. An evaluation using the chemically plausible extreme values for the $M_{\mathrm{IC}}$ (for GC peaks, the lowest and highest $M_{\mathrm{IC}}$ compatible with the observed retention times) may enable transformation to common units. When the assumed values for any $M_{\mathrm{IC}}$ of an unidentified IC significantly affects (relative to fitness for purpose) the transform values, then the ICs should be further characterized.

\subsection{Detection-Limited Quantitative Values}

When a critical or suspected IC cannot be confirmed as present using a method having fitfor-purpose sensitivity, an appropriate quantitative statement of the amount of the non-detected IC, ndIC, in the material is " $\leq \mathrm{LoD}$ ", where $\mathrm{LoD}$ represents the approximate "limit of detection" for the IC. The true amount of a particular ndIC in the material, $n_{\text {ndIC, }}$ is constrained to be between zero and LoD. The total amount of all ndICs in the material, $\sum n_{\mathrm{ndIC}}$, is constrained to be between zero and the sum of the individual LoDs, $\sum$ LoD.

In the purity analysis of elemental RMs, it has been traditional to estimate $\sum n_{\mathrm{ndIC}}$ as equal to one-half of $\sum$ LoD. While this is philosophically incorrect [81], it is perhaps defensibly conservative for elemental RMs given that there are fewer than 100 elemental ICs. Given the essentially infinite number of ICs that are plausibly in most organic materials, this estimate for $\sum n_{\text {ndIC }}$ will grow without bound as the search for ICs becomes more diligent.

There has been considerable discussion about how best to define LoD $[82,83]$. However, routine LoD values are seldom more than rough estimates of the amount of IC at which "If it were there, I'm pretty sure I would have seen it." Rigorous statistical evaluation of such "guesstimates" is neither practical nor necessary [83]. If the LoD is defined as the approximate amount of the IC that would be detectable with $\approx 95 \%$ confidence, the distribution of confidence on $n_{\text {ndIC }}$ can be approximated as an exponential distribution parameterized to have $95 \%$ of its area between zero and LoD [81]. Perhaps counter-intuitively, the maximum likelihood estimate of the location of such an exponential distribution is zero. That is, it is most likely that $\sum n_{\mathrm{ndIC}}=$ 0 .

The lower bound to the $95 \%$ confidence interval for $\sum n_{\text {ndIC }}, L B_{95}\left(\sum n_{\text {ndIC }}\right)$, is zero. The upper bound, $U B_{95}\left(\sum n_{\text {ndIC }}\right)$, can be approximated as the square root of the sum-of-the-squared LoD: 


$$
U B_{95}\left(\sum n_{\text {ndIC }}\right)=\sqrt{\sum_{i} \operatorname{LoD}_{i}^{2}}
$$

It should be noted that $U B_{95}\left(\sum n_{\text {ndIC }}\right)$ decreases with the diligence of the search for ICs. Before the presence of a particular IC is evaluated, the $U B_{95}$ (IC) (that is, the upper bound to the range of plausible values) is much larger than LoD. Choosing not to determine whether a given IC is present at a detectable level does not eliminate the uncertainty in whether it actually is present. On a less philosophical level, summing in quadrature ensures that $U B_{95}\left(\sum n_{\text {ndIC }}\right)$ grows only slowly with the number of explicitly included ndICs as long as the LoDs have similar magnitude.

Under the assumption of exponential distributions for the ndICs, the expected value for the sum of the amounts of detected and the non-detected components, $Y+\sum n_{\text {ndIC }}$, is just $Y$, since $\sum n_{\text {ndIC }}=0$. The uncertainty interval for $Y+\sum n_{\text {ndIC }}$ is asymmetric. It is defined by the lower and upper bounds:

$$
\begin{aligned}
& L B_{95}\left(\mathrm{Y}+\Sigma n_{\text {ndIC }}\right)=Y-\sqrt{U_{95}^{2}(Y)+L B_{95}^{2}\left(\Sigma n_{\text {ndIC }}\right)}=Y-U_{95}(Y) \\
& U B_{95}\left(\mathrm{Y}+\Sigma n_{\text {ndIC }}\right)=Y+\sqrt{U_{95}^{2}(Y)+U B_{95}^{2}\left(\Sigma n_{\text {ndIC }}\right)}
\end{aligned} .
$$

If both $U B_{95}\left(\sum n_{\mathrm{ndiC}}\right)$ and $U_{95}(Y)$ are small relative to fitness for purpose and a symmetric uncertainty is desired, then the $L B_{95}\left(Y+\sum n_{\text {ndIC }}\right)$ can be treated as if it were a symmetric $U_{95}\left(Y+\sum n_{\text {ndiC }}\right)$. If $U B_{95}\left(\sum n_{\text {ndIC }}\right)$ is less than about $30 \%$ of $U_{95}(Y)$, then the $U B_{95}\left(\sum n_{\text {ndIC }}\right)$ will contribute less than $10 \%$ of the combined uncertainty and ignoring the asymmetry will have very little penalty.

\subsection{Dealing with Boundary Violations}

The true values for any $x_{\mathrm{C}}$ or $w_{\mathrm{C}}$ are, by definition, constrained to be no less than 0 and no greater than 1 ; i.e., they are constrained to be within the interval $[0,1]$. However, because of the simplifying assumption that uncertainties are normally distributed, not infrequently a $\left\{x_{\mathrm{C}}, U_{95}\left(x_{\mathrm{C}}\right)\right\}$ or $\left\{w_{\mathrm{C}}, U_{95}\left(w_{\mathrm{C}}\right)\right\}$ extends below 0 or greater than 1 (hopefully, not both at the same time!). Rather than attempting a rigorous evaluation of the true shape of the uncertainty distribution for all such measurands and combining them in some complicated manner, the following Bayesian-inspired bias-correction and uncertainty expansion procedure has been suggested for practical use [83, 84].

- Combine all $\left\{x_{\mathrm{C}}, U_{95}\left(x_{\mathrm{C}}\right)\right\}$ or $\left\{w_{\mathrm{C}}, U_{95}\left(w_{\mathrm{C}}\right)\right\}$ necessary to get a particular result, $\left\{Y, U_{95}(Y)\right\}$, without regard to the $[0,1]$ boundaries.

- If $Y-U_{95}(Y)$ is less than 0 , define an asymmetric interval about $Y$ from $L B_{95}(Y)=0$ to $U B_{95}(Y)$ that will contain the plausible value of $Y$ with about $95 \%$ confidence. The $U B_{95}(Y)$ is determined by finding the smallest $\alpha$ such that 


$$
\frac{\int_{-\infty}^{\infty} N\left(y, Y, \frac{U_{95}(Y)}{2}\right) \partial y}{\int_{-\infty}^{\infty} N\left(y, Y, \frac{U_{95}(Y)}{2}\right) \partial y} \geq(1-0.05 / 2)
$$

where $N\left(z, Y, U_{95}(Y) / 2\right)$ is the probability density for a normal distribution of mean = $Y$, standard deviation $=U_{95}(Y) / 2$, evaluated over $y$. (This appears much more complex than it really is: the renormalization of the standardized normal cumulative distribution function by its truncated area and subsequent determination of $\alpha$ is easily accomplished using any table of - or spreadsheet function for - the cumulative normal.)

- If $Y+U_{95}(Y)$ is greater than 1 , define an asymmetric interval about $Y$ from $L B_{95}(Y)$ to $U B_{95}(Y)=1$ that will contain the plausible value of $Y$ with about $95 \%$ confidence. The $L B_{95}(Y)$ is determined by finding the smallest $\alpha$ such that

$$
\frac{\int_{-\infty}^{\infty} N\left(y, Y, \frac{U_{95}(Y)}{2}\right) \partial y}{\int_{-\infty}^{\infty} N\left(y, Y, \frac{U_{95}(Y)}{2}\right) \partial y} \leq(0.05 / 2) .
$$

- If $Y$ is less than 0 , set it to 0 ; if $Y$ is greater than 1 , set it to 1 .

- The $\approx 95 \%$ interval confidence interval about $Y$ is the asymmetric interval defined by the lower and upper bounds, $L B_{95}(Y) \leq Y \leq U B_{95}(Y)$. This asymmetric uncertainty can be reported as $Y_{-\left(Y-L B_{95}(Y)\right)}^{+\left(U B_{99}(Y) Y\right)}[19]$.

If a direct estimate of $\Sigma x_{\text {IC }}$ or $\sum w_{\text {IC }}$ is available (e.g., by DSC or \%GC-FID), then the upper boundary should be $\left(1-\Sigma x_{\mathrm{IC}}\right)+. U_{95}\left(1-\Sigma x_{\mathrm{IC}}\right)$ or $\left(1-\Sigma w_{\mathrm{IC}}\right)+U_{95}\left(1-\Sigma w_{\mathrm{IC}}\right)$ rather than 1 . 


\section{AN APPROACH TO PURITY ASSESSMENT}

"... [T]he determination of purity can never be a standardized procedure" [9]. The methods and rigor of analysis necessary and appropriate for a given neat-material RM depend upon the physical and chemical properties of the PC and ICs of the material and the purpose(s) for which the RM will be used.

The following outline presents a general approach to the assessment of purity that should provide chemically appropriate estimates of the amount of substance for the primary component and relevant impurity components. In conjunction with the basic requirements discussed in Section 3, following the spirit of this approach will ensure that the results of a purity assessment are recognized as sound chemical metrology.

Note that not all of the steps below are necessary or appropriate for all materials. Many of the measurements required can be accomplished using the same spectral or chromatographic analyses.

1) Is the material fit for purpose?

a. Examine the material for gross inhomogeneity, discoloration, suspended particulates, insoluble solids, and other potential problems that can be recognized from visual inspection and inexpensive assays such as dissolution, filtration, melting- and boiling-point determinations, refractive index, and mass loss on drying. Is the material plausibly fit for purpose? If not, can it be made so?

b. Confirm the identity of the PC.

c. If practical, determine $1-\sum x_{\mathrm{IC}}$ by DSC. Is this upper-bound estimate of $x_{\mathrm{PC}}$ sufficiently large for the material to be fit for purpose?

d. If practical, directly determine $w_{\mathrm{PC}}$ with an instrumentally augmented gravimetric and/or titrimetric method. If these recognized classical methods are inappropriate, analyze with qNMR on a high-field instrument or other appropriate direct method. Is the $\left\{w_{\mathrm{PC}}, U_{95}\left(w_{\mathrm{PC}}\right)\right\}$ sufficiently large for the material to be fit for purpose?

e. Evaluate any critical ICs using whatever method(s) will ensure that they are not present at levels that would render the material unfit for purpose. If detected, evaluate $\left\{w_{\mathrm{IC}}, U_{95}\left(w_{\mathrm{IC}}\right)\right\}$; if not detected, estimate $U B_{95}\left(w_{\mathrm{IC}}\right)$. Are the $\left\{w_{\mathrm{IC}}, U_{95}\left(w_{\mathrm{IC}}\right)\right\}$ sufficiently small for the material to be fit for purpose?

2) If it is (likely to be) fit for purpose, attempt to identify the ICs.

a. Assay/survey organic impurities (including all organic solvents used in the preparation or purification of the material).

i. Identify all peaks that appear in ${ }^{1} \mathrm{H}$ - and ${ }^{13} \mathrm{C}-\mathrm{NMR}$ (and ${ }^{19} \mathrm{~F}$ - and ${ }^{31} \mathrm{P}-\mathrm{NMR}$ if appropriate) spectra. Based on the identified structures, evaluate $\left\{w_{\mathrm{IC}}, U_{95}\left(w_{\mathrm{IC}}\right)\right\}$ using as many different transitions as practical. 
ii. Identify all peaks that appear in GC-FID (and other appropriate highsensitivity detection systems such as MS, AED, and EC) chromatograms. Based on the molecular structures and calculated or measured response factors, evaluate $\left\{w_{\mathrm{IC}}, U_{95}\left(w_{\mathrm{IC}}\right)\right\}$ using two or more different optimized but dissimilar separation systems.

iii. Identify all peaks that appear in LC- UV/Vis absorbance and other appropriate high sensitivity detection systems such as LC-Fl and LC-electron capture chromatograms. Based on the molecular structures and calculated or measured response factors, evaluate $\left\{w_{\mathrm{IC}}, U_{95}\left(w_{\mathrm{IC}}\right)\right\}$ using two or more different optimized but dissimilar separation systems.

b. Assay/survey inorganic impurities.

i. If water is a plausible impurity, determine $\left\{w_{\mathrm{H} 2 \mathrm{O}}, U_{95}\left(w_{\mathrm{H} 2 \mathrm{O}}\right)\right\}$ with a fit-forpurpose method such as thermogravimetry, Karl Fischer titration, or qNMR.

ii. Determine $\left\{w_{\mathrm{IC}}, U_{95}\left(w_{\mathrm{IC}}\right)\right\}$ for any suspected inorganic ICs with fit-forpurpose method(s). Ashing has limited sensitivity but may be useful for estimating a lower bound on total inorganics. High-sensitivity, multielement survey technique(s) such as X-ray fluorescence or ICP-MS should be used when significant levels of inorganic ICs are anticipated.

c. If not all suspected ICs have been detected, ensure that at least one of the methods used for general characterization was adequately sensitive for each missing IC. If necessary, conduct a specific search using adequately sensitive method(s). If detected, evaluate $\left\{w_{\mathrm{IC}}, U_{95}\left(w_{\mathrm{IC}}\right)\right\}$; if not detected, estimate $U B_{95}\left(w_{\mathrm{IC}}\right)$.

3) In light of ICs identified (particularly any contaminant components), evaluate whether the composition of the material is compatible with the known history and properties of the material. If it is not compatible, evaluate whether the search for contaminant ICs is sufficiently complete.

4) Calculate $\left\{1-\sum w_{\mathrm{IC}}, U_{95}\left(1-\sum w_{\mathrm{IC}}\right)\right\}$. Do the confidence intervals overlap with those of the direct determination of $w_{\mathrm{PC}}$ ? If they do not overlap and the difference between the direct and indirect estimates is significant relative to fit-for-purpose total uncertainty, search for the source(s) of the disagreement and rectify.

5) Once the direct $\left\{w_{\mathrm{PC}}, U_{95}\left(w_{\mathrm{PC}}\right)\right\}$ and indirect $\left\{1-\sum w_{\mathrm{IC}}, U_{95}\left(1-\sum w_{\mathrm{IC}}\right)\right\}$ purity estimates have been determined to describe the same measurand, combine them as in Section 5.1. The final value and its $95 \%$ confidence interval should be chemically consistent with all available information. 


\section{CASE STUDIES}

The following examples illustrate the general approach to purity assessment advocated in this document. The CRMs cited here were produced by several different providers and were selected for discussion partly because their certificates provided sufficient background information and analytical detail to enable independent evaluation of fitness-for-purpose beyond the scope of their originally stated need. While all of the CRMs discussed are fit for their original purpose(s), some have analysis flaws that impact the acceptability of their certification under current metrological practice. The techniques and extent of provided information may not reflect the providers' current practice.

\subsection{SRM 938 4-Nitrophenol}

Purpose: "This [material] is intended primarily for use in calibrating spectrophotometers for clinical analysis in which 4-nitrophenol is the chromogenic compound that is formed" [85]. The certified value for this material is a specific absorbance. The material was not intended for use as an amount-of-substance content CRM.

\begin{tabular}{cccccc} 
Component & Method & Amount & $U_{95}$ (Amount) & Units \\
\cline { 1 - 1 } \cline { 5 - 6 } 4-nitrophenol & Titration with alkali & & 0.9975 & - & $\mathrm{g} / \mathrm{g}$ \\
2-nitrophenol & Paired-ion chromatography & $<0.001$ & - & $\mathrm{g} / \mathrm{g}$ \\
3-nitrophenol & Paired-ion chromatography & $<0.001$ & - & $\mathrm{g} / \mathrm{g}$ \\
Water & Coulometric Karl Fischer & 0.0007 & - & $\mathrm{g} / \mathrm{g}$
\end{tabular}

Data source: Reference [85]. In addition to these values, information on the general characterization of 4-nitrophenol from various commercial sources and treatment histories is given in Ref. 86. The material apparently was also analyzed by DSC and "several LC separations." However, specific values for the SRM material are not presented. The analyses were apparently performed between 1977 and 1979.

PC identity confirmation: ${ }^{1} \mathrm{H}-,{ }^{13} \mathrm{C}-$, and ${ }^{15} \mathrm{~N}-\mathrm{NMR}$.

Heterogeneity: "No significant differences among samples [single samples from 10 different bottles] were observed with either of these tests [alkali titration and Karl Fischer]." [85] No sample size is specified.

Material history: Commercially obtained material, purified by twice recrystallizing it from water, once from dichloromethane, followed by a third recrystallization from water. The material was dried for several days, then sublimed under specified conditions.

Assessment: While this material was not intended for use as a purity CRM, the procedures used to prepare and evaluate it are exemplary - had the uncertainties of the various analyses performed been evaluated. Without these uncertainties, the purity value assigned to this material is not traceable nor can its fitness for purpose as a purity standard be assessed. 


\subsection{SRM 998 Angiotensin I (Human)}

Purpose: "This [material] is certified as a chemical of known purity. It is intended primarily for use in the calibration and standardization of procedures of the renin assay and as a reference peptide for amino acid analysis and high-performance liquid chromatography (HPLC)." [87]

\begin{tabular}{|c|c|c|c|c|}
\hline Component & Method & Amount & $u$ (Amount) & Units \\
\hline Angi & Amino acid a & 0.941 & 0.009 & $\mathrm{~g} / \mathrm{g}$ \\
\hline $\begin{array}{c}\text { Acetate } \\
\text { Peptide impurities } \\
\text { Non-peptide impurities }\end{array}$ & $\begin{array}{c}{ }^{1} \mathrm{H}-\mathrm{NMR} \\
\text { LC-UV/Vis }{ }_{215,280},{ }^{1} \mathrm{H}-\mathrm{NMR} \\
\text { LC-UV/Vis }{ }_{215},{ }^{1} \mathrm{H}-\mathrm{NMR}, \mathrm{MS}\end{array}$ & $\begin{array}{l}0.063 \\
\text { None } \\
\text { Traces }\end{array}$ & 0.002 & $\mathrm{~g} / \mathrm{g}$ \\
\hline
\end{tabular}

Data source: Reference [87]. The analyses were performed prior to 1983.

PC identity confirmation: LC-UV/Vis 215,280 with amino acid assay of all detected peaks.

Heterogeneity: "The calculated coefficient of variation between ampoules was $2.9 \%$." [87]

Material history: Commercially obtained material.

Assessment: The primary method of analysis used for this material was the indirect LCUV/Vis demonstration of a single peptide component with two mobile phases followed by a standard-additions GC assay of the component amino acids. The amount of the known acetate counter ion "impurity" was established by ${ }^{1} \mathrm{H}-\mathrm{NMR}$. No other non-peptide impurities were found at any but trace levels using LC, NMR, and MS analyses.

The certificate does not combine the $w_{\text {Amgiotensin I }}$ value with that obtained from $1-w_{\text {acetate }}$. Using the conventional coverage factor of 2 to expand the stated standard deviations, the indicated $\left\{w_{\text {amgiotensin }}, U_{95}\left(w_{\text {angiotensin }}\right)\right\}$ is $0.939 \pm 0.010$.

However, the ability of the LC assay to differentiate peptide impurities closely related to angiotensin I is discussed in supporting documents but not in the Certificate. A supporting document suggests that an isomeric form of the material may form during aging at $4{ }^{\circ} \mathrm{C}$; the certificate does not present evidence for the stability of the material at the recommended storage temperature of $-20^{\circ} \mathrm{C}$. The certificate does not describe the standard used in the standards addition assay nor the authority for a specific molar absorbance essential to the assay. No direct assay of total moisture is presented. Lacking complete evaluation of suspected impurities and without adequate documentation of calibration materials, the purity value assigned to this material is not traceable. 


\subsection{SRM 910 Sodium Pyruvate}

Purpose: "This [material] is certified as a chemical of known purity. It is intended primarily for use in the calibration and standardization of procedures for pyruvate, lactic dehydrogenase, and glutamic-pyruvic transaminase determinations in clinical analyses, and for critical evaluation of the routine working or secondary reference materials used in these procedures." [88]

\begin{tabular}{|c|c|c|c|c|}
\hline Component & Method & Amount & $u($ Amount $)$ & Units \\
\hline Sodium pyruvate & LC-UV/Vis 254 & 0.9850 & 0.0003 & $\mathrm{~g} / \mathrm{g}$ \\
\hline Sodium pyruvate & ${ }^{1} \mathrm{H}-\mathrm{NMR}$ & 0.9883 & 0.0005 & $\mathrm{~g} / \mathrm{g}$ \\
\hline & Certified & 0.987 & 0.002 & $\mathrm{~g} / \mathrm{g}$ \\
\hline Parapyruvate & LC-UV/Vis 254 & 0.0102 & 0.0003 & $g / g$ \\
\hline Parapyruvate & ${ }^{1} \mathrm{H}-\mathrm{NMR}$ & 0.0068 & 0.0005 & $\mathrm{~g} / \mathrm{g}$ \\
\hline & Certified & 0.009 & 0.002 & $\mathrm{~g} / \mathrm{g}$ \\
\hline Methanol & ${ }^{1} \mathrm{H}-\mathrm{NMR}$ & 0.0021 & - & $g / g$ \\
\hline Pyruvate oligomers & LC-UV/Vis $_{254}$ & $<0.005$ & - & $\mathrm{g} / \mathrm{g}$ \\
\hline Organic impurities other than & ${ }^{13} \mathrm{C}-\mathrm{NMR}$ & $<0.006$ & - & $\mathrm{mol} / \mathrm{mol}$ \\
\hline Moisture & ${ }^{1} \mathrm{H}-\mathrm{NMR} ?$ & 0.0028 & - & $g / g$ \\
\hline Water-insoluble matter & ? & 0.00004 & - & $\mathrm{g} / \mathrm{g}$ \\
\hline
\end{tabular}

Data source: Reference [88]. The analyses were performed prior to 1981.

PC identity confirmation: ${ }^{1} \mathrm{H}-\mathrm{NMR},{ }^{13} \mathrm{C}-\mathrm{NMR}$, and elemental analysis.

Heterogeneity: "The homogeneity of the SRM, as determined by liquid chromatography and proton and ${ }^{13} \mathrm{C}$ NMR, was found to be satisfactory." [88]

Material history: Commercially obtained material.

Assessment: The impurity assessment of this material is exemplary. All ICs detected by LCUV/Vis were characterized by ${ }^{1} \mathrm{H}$ - and ${ }^{13} \mathrm{C}-\mathrm{NMR} .{ }^{13} \mathrm{C}-\mathrm{NMR}$ was used to establish an upper bound on the total amount of uncharacterized organic ICs that is smaller than the uncertainties in the analysis of the identified components. The stabilities of these impurities and of methanol to material handling were demonstrated.

The certificate does not combine the various results for the ICs nor compare the $w_{\text {pyruvate }}$ values with an estimate of $1-w_{\text {parapyruvate }}-\sum w_{\mathrm{IC}}$. Using the conventional coverage factor of 2 to expand the stated standard deviations, the indicated $\left\{w_{\text {pyruvate }}, U_{95}\left(w_{\text {pyruvate }}\right)\right\}$ is $0.986 \pm 0.002$.

However, little description is provided on the methods used for moisture, water-insoluble matter, and elemental composition. More critically, the LC and qNMR methods are incompletely described. While spectroscopic response factors for pyruvate and parapyruvate are presented, there is no description of the materials used in their evaluation. Neither the basic approach nor sufficient experimental details are specified to establish the fitness-for-purpose of the qNMR evaluations. Without the requisite auxiliary information, the purity value assigned to this material is not traceable nor can its fitness for purpose as a purity standard be assessed. 


\subsection{LGC1110 (pp'-Dichlorodiphenyl)dichloroethylene (pp'-DDE)}

Purpose: "This material is intended for use in the preparation of solutions for the calibration of analytical instruments used in pesticide residue and formulation analysis." [89]

\begin{tabular}{|c|c|c|c|c|}
\hline Component & Method & Amount & $\underline{U_{95}(\text { Amount })}$ & Units \\
\hline $1-\sum x_{I C}$ & $\mathrm{DSC}$ & 0.9974 & 0.0006 & $\mathrm{~mol} / \mathrm{mol}$ \\
\hline $1-\sum w_{\mathrm{IC}}$ & \%area GC-FID & 0.9948 & 0.0004 & $\approx \mathrm{g} / \mathrm{g}$ \\
\hline $1-\Sigma w_{\mathrm{IC}}$ & \%area LC-UV/Vis 248 & 0.9966 & 0.0008 & ? \\
\hline Water & Karl Fischer & 0.00010 & 0.00008 & $g / g$ \\
\hline pp'-DDE & Combined & 0.996 & 0.004 & $g / g$ \\
\hline
\end{tabular}

Data source: Reference [90]. Some additional information is supplied in Reference [57] where this material is used as an example of a neat-substance CRM. The analyses were performed prior to 1997.

PC identity confirmation: Not stated.

Heterogeneity: "The material was assessed on the basis of six randomly selected 2-mg portions. The material was judged to be homogenous as the variation between portions was not significantly different from the analytical variation." [90]

Material history: Commercially obtained material of $99 \%$ nominal purity, ground, sieved to $\leq 710 \mu \mathrm{m}$, and dried under vacuum.

Assessment: With the exception of water, none of the ICs are identified. The low amount of water and of $\Sigma w_{\text {IC }}$ observed suggests that the $1-\Sigma x_{\text {IC }}$ DSC results are valid estimates of $1-\Sigma w_{\text {IC }}$. However, the DSC results represent only an upper bound to purity and the LC-UV/Vis results are equivocal without supporting evidence on the nature of the non-water ICs. Thus, the "best" available evidence is that, with $95 \%$ confidence, $0.9948-0.0004 \leq w_{\mathrm{pp}}$-DDE $\leq 0.9974+0.0006$ or, equivalently, $w_{\mathrm{pp} \text { '-DDE }}=0.9948_{-0.0004}^{+0.0032}$. Since the DSC $x_{\mathrm{pp} \text { '-DDE }}$ is larger than the \%GC-FID $w_{\mathrm{pp}}$-DDE, traceability is primarily based upon the validity of the \%GC-FID assumptions. 


\subsection{Arsenobetaine Master Material for BCR-626}

Purpose: High-purity arsenobetaine was prepared, characterized, and used to prepare the solution standard BCR-626 [91]. BCR-626 is intended for use as a calibrant in arsenic speciation studies; the master material used in the preparation of BCR-626 is not available.

\begin{tabular}{|c|c|c|c|c|}
\hline Component & Method & Amount & $U_{95}$ (Amount) & Units \\
\hline Organic $\Sigma w_{\mathrm{IC}}$ & $\begin{array}{c}{ }^{1} \mathrm{H}-\mathrm{NMR}, \mathrm{GC}-\mathrm{FID} \text {, fast atom } \\
\text { bombardment-MS }\end{array}$ & $\overline{<0.0001}$ & & $\mathrm{~g} / \mathrm{g}$ \\
\hline Metal $\Sigma w_{\text {IC }}$ & $\begin{array}{l}\text { ICP-OES, energy dispersive X-ray } \\
\text { fluorescence }\end{array}$ & $<0.00001$ & & $\mathrm{~g} / \mathrm{g}$ \\
\hline Arsenic $\Sigma w_{\mathrm{IC}}$ & HPLC-ICP-MS & $<0.0015$ & & $\mathrm{~g} / \mathrm{g}$ \\
\hline Classical ions $\Sigma w_{\mathrm{IC}}$ & $\begin{array}{l}\text { Capillary zone electrophoresis with } \\
\text { UV detection }\end{array}$ & $<0.002$ & & $g / g$ \\
\hline Total C & ICP-OES & 0.334 & 0.003 & $\mathrm{~g} / \mathrm{g}$ \\
\hline Total H & ICP-OES & 0.0625 & 0.0007 & $\mathrm{~g} / \mathrm{g}$ \\
\hline Total As & ICP-OES & 0.418 & 0.008 & $\mathrm{~g} / \mathrm{g}$ \\
\hline $\mathrm{H}_{2} \mathrm{O}$ & Thermogravimet & 0.023 & Not & $g / g$ \\
\hline Worst case $\mathrm{H}_{2} \mathrm{O}$ & Expected C - Total C & 0.009 & Not stated & $\mathrm{g} / \mathrm{g}$ \\
\hline Arsenobetaine & Combined & 0.997 & 0.006 & $g / g$ \\
\hline
\end{tabular}

Data source: Reference [91]. The analyses were performed prior to 1997.

PC identity confirmation: Method of preparation and ${ }^{1} \mathrm{H}-\mathrm{NMR}$.

Heterogeneity: Total As was assayed by ICP-OES in all of the intermediate "mother solutions" prepared from the master material and in the water-solution CRM units prepared from the mother solutions. Total analysis repeatability for both materials was $0.6 \%$.

Material history: The material was synthesized and purified according to stated procedures [91].

Observations: The pilot laboratories made a thorough search for ICs. The material was recognized as very hygroscopic, but moisture content was not well evaluated in material protected from ambient humidity. Rather than the authors' focus on just the difference between the observed and calculated total $\mathrm{C}$, least square minimization of the total $\mathrm{C}, \mathrm{H}$, and As results suggests that $\left\{w_{\mathrm{H} 2 \mathrm{O}}, U_{95}\left(w_{\mathrm{H} 2 \mathrm{O}}\right)\right\}$ for the material is $\{0.007, \pm 0.017\}$ rather than the stated "worst case" of 0.009 . While better information on water is needed for a believable combination, based on complete analysis of supplied data the certified value would be better stated as $0.993_{-0.018}^{+0.007}$. Traceability is no stronger than its weakest link; in this case, moisture determination. 


\subsection{BCR 289 2,4'-Dichlorobiphenyl (PCB-8)}

Purpose: “... intended mainly for the qualitative and quantitative calibration of analytical apparatus and methods (determination of retention times, response factors and reference spectra in chromatographic and spectroscopic analysis) and for the study of biological activity." [92]

$\frac{\text { Component }}{\text { Organic } \Sigma w_{\mathrm{IC}}} \frac{\text { Method }}{\begin{array}{c}23 \text { determinations by } 8 \text { laboratories using } \\ \text { GC-FID, GC-MS, and LC-UV/Vis. }\end{array}} \frac{\text { Amount }}{0.0034} \frac{L B_{95}}{0.0025} \frac{U B_{95}}{0.0058} \frac{\text { Units }}{\mathrm{g} / \mathrm{g}}$

Tentative identification of ICs consistent

with being other $\mathrm{Cl}_{2}$-biphenyl isomers.

\begin{tabular}{|c|c|c|c|c|c|}
\hline Inorganic $\Sigma w_{\mathrm{IC}}$ & Ashing of HF-digest & 0.0003 & 0.0000 & 0.0006 & $\mathrm{~g} / \mathrm{g}$ \\
\hline Total $\Sigma w_{\text {IC }}$ & Combined & 0.0037 & 0.0025 & 0.0064 & $\mathrm{~g} / \mathrm{g}$ \\
\hline PCB-8 & $1-$ Total $\Sigma w_{\mathrm{IC}}$ & 0.9963 & 0.9936 & 0.9975 & $g / g$ \\
\hline
\end{tabular}

Data source: Reference [92]. The analyses were performed prior to 1987.

PC identity confirmation: Method of preparation and IR spectroscopy, melting point, GC retention time, and X-ray diffraction structure determination.

Heterogeneity: "The between-bottle homogeneity was assessed [using an undefined LC method] on six vials taken at random ... [the result] was not significantly different from that of a replicate analysis of a single solution. No within-bottle study was undertaken because previous studies have shown that the homogenization process used in the preparative phase was fully effective." [92]

Material history: The material was synthesized and purified according to published procedures [92].

Observations: This material was evaluated as part of a multi-laboratory, multi-method investigation. In aggregate, the large number of differing GC and LC analyses provide reasonable evidence of a complete analysis. The asymmetric confidence bounds result from the authors' assumption that the individual organic $\Sigma w_{\text {IC }}$ were log-normally distributed. The results are adequately traceable. 


\subsection{NMIJ CRM 4011-a o-Xylene}

Purpose: “...for use in calibration of analytical instruments and other applications.” [93]

\begin{tabular}{|c|c|c|c|c|}
\hline Component & Method & Amount & $U_{95}$ (Amount) & Units \\
\hline $1-\Sigma x_{\mathrm{IC}}$ & Adiabatic calorimetry & 0.99933 & 0.00002 & $\mathrm{~mol} / \mathrm{mol}$ \\
\hline$n$-nonane & GC-FID/MS & 0.0000482 & 0.0000024 & $g / g$ \\
\hline$p$-xylene & GC-FID/MS & 0.0000394 & 0.0000038 & $g / g$ \\
\hline$m$-xylene & GC-FID/MS & 0.000220 & 0.000020 & $\mathrm{~g} / \mathrm{g}$ \\
\hline Isopropylbenzene & GC-FID/MS & 0.000134 & 0.000006 & $\mathrm{~g} / \mathrm{g}$ \\
\hline Water & Coulometric Karl Fischer & 0.000015 & 0.000004 & $\mathrm{~g} / \mathrm{g}$ \\
\hline Inorganics & ICP-MS & Not stated & Not stated & $\mathrm{g} / \mathrm{g}$ \\
\hline Consecutive $1-\Sigma x_{\mathrm{IC}}$ & Combined & 0.999543 & 0.000022 & $g / g$ \\
\hline$o$-xylene & Combined & 0.99940 & 0.00003 & $g / g$ \\
\hline
\end{tabular}

Data source: Reference [93]. The analyses were performed prior to 2002.

PC identity confirmation: Not stated.

Heterogeneity: \%Area GC-FID and water assay by Karl-Fischer: "less than uncertainty of purity determination" [93].

Material history: Commercially obtained special-grade reagent, dehydrated, double-distilled, and ampouled into dark brown hard glass under argon.

Observations: Only the calorimetric $1-\Sigma x_{\text {IC }}$ is certified, limiting the formal traceability to the validity of the adiabatic calorimetric method used. However, all of the observed ICs are compatible with material history and the search for contaminants appears adequate. The assumption that the $1-\Sigma x_{\text {IC }}=1-\Sigma w_{\text {IC }}$ is not defended but the IC levels are sufficiently small that it appears valid. While there is no overlap between the direct $\{0.99933, \pm 0.00002\}$ and consecutive $\{0.99954, \pm 0.00002\}$ estimates of $1-\Sigma w_{\mathrm{IC}}$, the calorimetric estimate is somewhat smaller and therefore quite believable as an estimate of the true amount of all impurities. The combined estimate of $\{0.99940, \pm 0.00003\}$ provided as a reference value thus unnecessarily combines the direct and consecutive determinations, but would be validly traceable except for the uncertainty being too small: application of Equation 6 to the two independent determinations yields a value of $\{0.9994, \pm 0.0003\}$. 


\subsection{Technical Profenofos}

Purpose: Technical-grade O-(4-bromo-2-chlorophenyl) O-ethyl S-propyl phosphorothioate (profenofos) insecticide was analyzed as part of a project to demonstrate the utility of qNMR techniques for the direct determination of chemical purity. The material was not intended nor is it (unfortunately) available for use as an RM [41].

\begin{tabular}{|c|c|c|c|c|}
\hline Component & Method & Amount & $u$ (Amount) & Units \\
\hline Profenofos & ${ }^{1} \mathrm{H}-\mathrm{NMR}$ & 0.9463 & 0.0031 & $\mathrm{~g} / \mathrm{g}$ \\
\hline Profenofos & ${ }^{31} \mathrm{P}-\mathrm{NMR}$ & 0.9461 & 0.0065 & $g / g$ \\
\hline
\end{tabular}

Data source: Reference [41]. The analyses were performed prior to 2002.

PC identity confirmation: ${ }^{1} \mathrm{H}$ - and ${ }^{31}$-P NMR.

Heterogeneity: Within-batch heterogeneity is included in material uncertainty evaluations.

Material history: Commercially obtained material.

Observations: The qNMR analyses of this material are exemplary. All procedures and standards are identified, all NMR signals are accounted for, and a complete evaluation of sources of uncertainty is provided. NMR-active ICs are identified and shown to be compatible with the synthetic route. The indicated $\left\{w_{\text {profenofos, }} U_{95}\left(w_{\text {profenofos }}\right)\right\}$ value of $0.946 \pm 0.007$ is fully traceable to the qNMR standards used. Material stability, evaluation of the NMR-active ICs, and a survey for NMR-inactive ICs would be the only additional information required to enable determination of this material's fitness-for-purpose as an RM. 


\subsection{SRM 911b Cholesterol}

Purpose: "This [material] is primarily for use in the calibration and standardization of procedures for the determination of cholesterol in clinical samples and for routine evaluations of daily working standards used in these procedures." [94]

\begin{tabular}{|c|c|c|c|c|}
\hline Component & Method & Amount & $U_{95}$ (Amount) & Units \\
\hline Direct $\Sigma x_{\mathrm{IC}}$ & $\mathrm{DSC}$ & 0.00095 & 0.00039 & $\mathrm{~mol} / \mathrm{mol}$ \\
\hline 7-Dehydrocholesterol & GC-MS & 0.0003 & & $g / g$ \\
\hline Campesterol & GC-MS & 0.0005 & & $g / g$ \\
\hline Sitosterol & GC-MS & 0.0005 & & $g / g$ \\
\hline Lathosterol & GC-MS & $\leq 0.0002$ & & $\mathrm{~g} / \mathrm{g}$ \\
\hline Cholestanol & GC-MS & $\leq 0.0002$ & & $\mathrm{~g} / \mathrm{g}$ \\
\hline Other steroids & GC-MS & $\leq 0.0001$ & & $\mathrm{~g} / \mathrm{g}$ \\
\hline Consecutive $\Sigma w_{\mathrm{IC}}$ & GC-MS & $\leq 0.0018$ & 0.0005 & $\mathrm{~g} / \mathrm{g}$ \\
\hline $\begin{array}{l}\text { 5,7,9(11)-trienes } \\
\text { Organic imnurities }\end{array}$ & $\begin{array}{c}\text { UV/Vis } \\
{ }^{1} \mathrm{H}-\text { and } \\
{ }^{13} \mathrm{C}-\mathrm{NMR}\end{array}$ & $\leq 0.0003$ & & $g / g$ \\
\hline Chlorine & NAA & 0.000006 & 0.000001 & $\begin{array}{l}g / g \\
g / g\end{array}$ \\
\hline Bromine & NAA & 0.000038 & 0.000007 & $\mathrm{~g} / \mathrm{g}$ \\
\hline Iodine & NAA & $<0.0000001$ & & $\mathrm{~g} / \mathrm{g}$ \\
\hline Volatiles & mass loss on drying & 0.00020 & 0.00001 & $g / g$ \\
\hline Insoluble matter & filtration & 0.00017 & & $g / g$ \\
\hline Ash & combustion & 0.00006 & & $\mathrm{~g} / \mathrm{g}$ \\
\hline Cholesterol & Combined & 0.998 & 0.001 & $g / g$ \\
\hline
\end{tabular}

Data source: Reference [94] and supporting Reports of Analysis. The analyses were performed from 1986 to 1988 .

PC identity confirmation: Multiple direct comparisons (including GC-MS, NMR, melting point, and optical rotation) with SRM 911 a; however, there is no explicit statement on record for the method of identity confirmation used for SRM 911a or its precursor SRM 911.

Heterogeneity: No significant heterogeneity, as " determined by GC-MS and DSC" [94].

Material history: Commercially obtained material that had been treated with bromine, dehalogenated with zinc and recrystallized from methanol to remove companion steroids [94].

Observations: Expected ICs, including inorganic and solvents as well as sterols, were identified and characterized. One observed steroid IC was quantitatively determined by GC-MS standard addition; all others were estimated from peak heights relative to the characterized IC. Contaminant ICs were excluded using NMR, UV/Vis, and infrared spectroscopies, thin-layer chromatography, and GC-MS. Value assignment was by chemical judgment, regarding the DSC result as an upper-bound and the sum of the expected ICs as a pessimistic lower-bound. Given the low IC levels, the implicit assumption that $\Sigma x_{\mathrm{IC}} \approx \Sigma w_{\mathrm{IC}}$ appears justified. The resulting certified purity, while stated with less precision and wider uncertainty than a more mathematical combination could provide, covers the chemically plausible values and is thus fully traceable. 


\section{OPPORTUNITIES}

There is little that is unique about the selection, production, analysis, and certification of neat materials relative to calibration solution and natural-matrix RMs. While no single chemical measurement technology is by itself fully "primary", a number of methods can be made "primary enough" when sufficiently validated for the specific determination and augmented by other measurements and diligent efforts to identify and quantify all impurity components that impact the material's fitness for purpose. We conclude that currently available analytical methods can adequately establish the SI traceability of amount of substance measurements of neat materials. However, several opportunities for improving traceability infrastructure have been identified.

1) Few of the CRMs that are of potential interest as qNMR comparators are suitably certified as molecular entities. For example, NIST SRM 1071b Triphenyl Phosphate (Standard for Determination of Phosphorus in Petroleum Products) is certified to be $\{9.48, \pm 0.08\}$ phosphorus "weight percent". This total phosphorus value does not provide a traceable link for use of this material as a source of $\left(\mathrm{C}_{6} \mathrm{H}_{5}\right)_{3} \mathrm{PO}_{4}$ for a ${ }^{1} \mathrm{H}-{ }^{13} \mathrm{C}$ - and ${ }^{31} \mathrm{P}$-qNMR standard. It may be possible to recertify some established neat-material CRMs for use with qNMR with little or no additional chemical characterization. A number of compounds have been proposed as qNMR comparators that are not currently available as neat-material CRMs. However, since qNMR compares nuclei and not molecules, it is likely that only a small suite of neat-material CRMs need to be recertified or developed to meet the traceability needs of this measurement technology [41].

2) Methods that directly determine the PC mole fraction, such as \%qNMR and \%GC, can provide highly repeatable results relatively quickly and inexpensively. However, there is little information regarding the quantitative evaluation of the uncertainties in such results. These uncertainty sources include: the uniformity of the response factor relating signal intensity to mole fraction for all components, the extent to which the total signal represents the totality of ICs, and the degree of isolation of signal from the PC from that of the ICs. General procedures for demonstrating the adequacy of the assumptions and evaluating their uncertainties would enhance the traceability of the results provided by these direct methods.

3) "Measurement-assisted" QSPRs that combine experimental determination of response factors for a limited set of structurally diverse compounds plus a few compounds similar to the desired IC could greatly improve the prediction of chromatographic detector response factors from molecular structure. Such QSPR predictions would improve the quantitative utility of \%GC and \%LC methods when exemplar materials for some ICs are too expensive or unavailable for experimental response factor determination.

4) A very sensitive, universal mass detector for $\mathrm{LC}$ would greatly facilitate the development of $\%$ LC methods for purity determination. IRMS and CRIMS, both of which quantitatively convert molecules to a set of small polyatomic species and enable characterization of the relative molecular mass of the original compound through the ratios of characteristic stable isotopes, may meet this need. LC-CRIMS can be used with conventional mass spectrometers and enables a more complete evaluation of a molecule's elemental composition. Neither technique has apparently been developed for use in purity evaluations. 


\section{ACKNOWLEDGMENTS}

The authors thank Robert W. Gerlach (Lockheed Martin Information Technology, Las Vegas, NV USA) and Stephen L.R. Ellison (LGC, Teddington, UK) for arguing over various issues concerning the combining of not-quite-the-same values. Their insights have been invaluable, but the current work does not necessarily reflect all of their views.

We thank Harald Jancke (BAM, Berlin, Germany) for his careful critique of the manuscript and for his guidance on qNMR techniques. We thank Tareq Saed Al Deen (University of New South Wales, Sydney, Australia) for making available data from his qNMR analyses of technical Profenofos.

The authors acknowledge the advice and generally gentle correction of their NIST colleagues: Richard G. Christensen, Pamela M. Chu, Gary W. Kramer, William A. MacCrehan, Kenneth W. Pratt, Michele M. Schantz, Katherine S. Sharpless, Robert Q. Thompson, John C. Travis, and Michael J. Welch. Bob Gerlach and Kathy Sharpless have done much to ensure that what we've written is what we actually meant. 


\section{REFERENCES}

1 BIPM. The International System of Units (SI), $7^{\text {th }}$ Edition. Paris, France (1998). http://www 1.bipm.org/en/si/si_brochure/

2 ISO/REMCO. ISO Guide 35 - Certification of reference materials: general and statistical principles. Third edition, Draft 2. ISO, Geneva, Switzerland (2001).

3 M. Stoeppler, W.R. Wolf, P.J. Jenks, eds. Reference Materials for Chemical Analysis. Certification, Availability and Proper Usage. Wiley-VCH, Weinheim, Germany (2001).

4 CITAC/EURACHEM. Guide to quality in analytical chemistry. An aid to accreditation. CITAC / EURACHEM 2002, pp. 1-57. http://www.eurachem.ul.pt/guides/CITAC\%20EURACHEM\%20GUIDE.pdf

5 L.A.K. Stavely, ed., The characterization of chemical purity. Organic compounds, International Union of Pure and Applied Chemistry, Physical Chemistry Division, Commission on Physicochemical Measurements and Standards, Butterworth \& Co. (Publishers) Ltd, London, UK (1971).

$6 \mathrm{BIPM/IEC/IFCC/ISO/IUPAC/IUPAP/OIML.} \mathrm{International} \mathrm{vocabulary} \mathrm{of} \mathrm{basic} \mathrm{and} \mathrm{general}$ terms in metrology, 2nd Edition. ISO, Geneva, Switzerland (1993).

7 D. Holcombe. Alphabetical index of defined terms and where they can be found. Part I: A-F, Accreditation and Quality Assurance 1999;4:525-530.

Part II: G-Q, Accreditation and Quality Assurance 2000;5:77-82.

Part III: R-Z, Accreditation and Quality Assurance 2000;5:159-164.

8 The Oxford English Dictionary, Oxford University Press 1971.

9 W.M. Smit. The concept of purity, and its bearing on methods used to characterize purity. In: L.A.K. Stavely, ed., The characterization of chemical purity. Organic compounds, International Union of Pure and Applied Chemistry, Physical Chemistry Division, Commission on Physicochemical Measurements and Standards, Butterworth \& Co. (Publishers) Ltd, London, UK (1971), pp. 7-10.

10 IUPAC, Compendium of analytical nomenclature, definitive rules 1997. ("Orange Book"), 3rd Edition. Blackwell, Oxford (1998).

11 R. Bethem, J. Boison, J. Gale, D. Heller, S. Lehotay, J. Loo, S. Musser, P. Price, and S. Stein. Establishing the fitness for purpose of mass spectrometric methods. J American Society for Mass Spectrometry 2003;14:528-541.

12 Eurachem, The fitness for purpose of analytical methods. A laboratory guide to method validation and related topics. First Internet Version, Dec. 1998 (1998). http://www.eurachem.ul.pt/ 
13 T. Fearn, S.A. Fisher, M. Thompson, and S.L.R. Ellison. A decision theory approach to fitness for purpose in analytical measurement, Analyst 2002;127:818-824.

14 P. De Bievre and H.S. Peiser. The reliability of values of molar mass, the factor that relates measurements expressed in two SI base units (mass and amount of substance), Metrologia 1997;34(1):49-59.

15 S.D Phillips, W.T. Estler, T. Doiron, K.R. Eberhardt, and M.S. Levenson. A careful consideration of the calibration concept, J Res Natl Inst Stan 2001;106(2):371-9.

16 EURACHEM/CITAC. Traceability in chemical measurement. A guide to achieving comparable results in chemical measurement. Voting Draft, March 2003, (2003). http://www.eurachem.ul.pt/

17 T.J. Quinn. Primary methods of measurement and primary standards, Metrologia 1997;34(1):61-65.

18 P. De Bievre and P.D.P Taylor. Traceability to the SI of amount-of-substance measurements: from ignoring to realizing, a chemist's view, Metrologia 1997;34(1):67-75.

19 ISO. Guide to the expression of uncertainty in measurement. ISO, Geneva, Switzerland, (1995).

20 ISO/IEC 17025. General requirements for the competence of testing and calibration laboratories, First Edition. ISO, Geneva, Switzerland (1999).

21 W. Wegscheider. Validation of analytical methods. In: H. Günzler, Acceditation and Quality Assurance in Analytical Chemistry, Springer, Berlin, Germany (1996), Chapter 6, pp. 135-158.

22 M.J.T. Milton, T.J. Quinn. Primary methods for the measurement of amount of substance. Metrologia 2001;38(4):289-296.

23 M.J.T. Milton. Clarifying the CCQM definition of a primary method of measurement. In: PIBM, Comité Consultatif pour la Quantité de Matière, Working documents of the 4th Meeting (February 1998) 1998;CCQM/98-5;1-7.

24 W.E. May, Achieving international comparability for chemical measurements: A myth or a milestone for the new millennium. International Forum on Frontiers in Science and Measurement. 21-25 June 1999, NPL, Teddington, England, UK.

25 ISO. Guide 34: General requirements for the competence of reference material producers. Second edition. ISO, Geneva, Switzerland, (2000).

26 ILAC. Guidelines for the requirements for the competence of reference material producers. ILAC-G12:2000, (2000). http://www.ilac.org 
27 W. May, R. Parris, C. Beck, J. Fassett, R. Greenberg, F. Guenther, G. Kramer, S. Wise, T. Gills, J. Colbert, R. Gettings, B. MacDonald. Definitions of terms and modes used at NIST for value-assignment of reference materials for chemical measurements. NIST Special Publication 260-136, NIST, Gaithersburg, MD USA (2000).

28 ISO. Guide 31: Reference materials - Contents of certificates and labels. ISO, Geneva, Switzerland, (2000).

29 I.M. Kolthoff. Treatise on analytical chemistry. Part I. Theory and Practice, edited by I.M. Kolthoff and Philip J. Elving, $1^{\text {st }}$ edition. Volumes 1 to 12 . Wiley, New York, NY USA Wiley, (1973 - 1978).

30 C.M. Beck II. Toward a revival of classical analysis. Metrologia 1997;34(1):19-30.

31 T.W. Vetter, K.W. Pratt, G.C. Turk, C.M. Beck II, and T.A. Butler. Using instrumental techniques to increase the accuracy of the gravimetric determination of sulfate. Analyst 1995;120:2025-2032.

32 T.W. Vetter, Gravimetric Analysis of Sodium in Serum. CCQM Primary Methods Symposium: How Far Does the Light Shine? 4-5 April 2000, BIPM, Sèvres, France.

33 B. King. Review of the potential of titrimetry as a primary method. Metrologia 1997;34(1):77-82.

34 M. Máriássy, Coulometry for Purity Determination. CCQM Primary Methods Symposium: How Far Does the Light Shine? 4-5 April 2000, BIPM, Sèvres, France.

35 H. Felber and S. Rezzonico, Titrimetry: Determination of Copper in a monoelemental standard. Symposium: How Far Does the Light Shine? 4-5 April 2000, BIPM, Sèvres, France.

$36 \mathrm{H}$. Jancke. NMR spectroscopy as a primary analytical method.- In: PIBM, Comité Consultatif pour la Quantité de Matière, Working documents of the 4th Meeting (February 1998) 1998;CCQM/98-2;1-12. See also: H. Jancke. NMR as a primary analytical method of measurement. Nachr Chem Tech Lab 1998;46(7-8):720-722.

37 D.L. Rabinstein and D.A. Keire, Quantitative Analysis by NMR. In: A.I. Popov and K. Hallenga, ed., Practical Spectroscopy, Vol. 11: Modern NMR Techniques and Their Application in Chemistry, M. Dekker, New York, NY, USA (1990), pp. 323-369.

38 R.F. Evilia, Quantitative NMR spectroscopy. Analytical Letters 2001;34(13):2227-2236.

39 G.F. Paul. qNMR - A versatile concept for the validation of natural product reference compounds, Phytochemical Analysis 2001;12(1):28-42. 
40 R.J. Wells, J.M. Hook, T.S. Al-Deen TS, and D.B. Hibbert. Quantitative nuclear magnetic resonance (QNMR) spectroscopy for assessing the purity of technical grade agrochemicals: 2,4-dichlorophenoxyacetic acid (2,4-D) and sodium 2,2-dichloropropionate (Dalapon sodium). J Agricultural and Food Chemistry 2002;50(12):3366-3374.

41 T.S. Al Deen, D.B. Hibbert, J.M. Hook, and R.J. Wells. Quantitative nuclear magnetic resonance spectrometry - II. Purity of phosphorus-based agrochemicals glyphosate (N(phosphonomethyl)-glycine) and profenofos (O-(4-bromo-2-chlorophenyl) O-ethyl S-propyl phosphorothioate) measured by H-1 and P-31 QNMR spectrometry. Analyical Chimica Acta 2002;474(1-2):125-135.

42 F. Kasler. Quantitative Analysis by NMR Spectroscopy. Academic Press, London, UK. (1973).

43 T.J. Henderson. Quantitative NMR Spectroscopy using coaxial inserts containing a reference standard: Purity determinations for military nerve agents. Analytical Chemistry 2002;74(1):191-198.

44 L. Griffiths and A.M. Irving. Assay by nuclear magnetic resonance spectroscopy: quantification limits. Analyst 1998;123:1061-1068.

45 Adapted from: K. Lee and W.A. Anderson. Nuclear Spins, Moments, and Magnetic Resonance Frequencies. In: CRC Handbook of Chemistry and Physics, $62^{\text {nd }}$ Edition, R.C. Weast and M.J. Astle, eds. CRC Press, Boca Raton, FL USA (1982), pp E-67 - E-71.

46 R.L. Watters, K.R. Eberhardt, E.S. Beary, and J.D. Fassett. Protocol for isotope dilution using inductively coupled plasma-mass spectrometry (ICP-MS) for the determination of inorganic elements. Metrologia 1997;34(1):87-96.

47 M.J.T. Milton and R.I. Wielgosz. Uncertainty in SI-traceable measurements of amount of substance by isotope dilution mass spectrometry. Metrologia 2000;37(3):199-206.

48 M. Welch, ID-GC/MS for Clinical Diagnostic Markers. P. De Bièvre, Absolute Isotopic Ratio Measurements for Determining Amount of Substance. CCQM Primary Methods Symposium: How Far Does the Light Shine? 4-5 April 2000, BIPM, Sèvres, France.

49 M.J.T. Milton and J.A. Wang. High accuracy method for isotope dilution mass spectrometry with application to the measurement of carbon dioxide. International Journal of Mass Spectrometry 2002;218(1):63-73.

50 J.T. Brenna, T.N. Corso, H.J. Tobias, and R.J. Caimi. High-precision continuous-flow isotope ratio mass spectrometry. Mass Spectrometry Reviews 1997;16:227-258.

51 F.P. Abramson. CRIMS: Chemical reaction interface mass spectrometry. Mass Spectrometry Reviews 1994;13:341-356.

52 R.D. van Zee, J.T. Hodges, and J.P. Looney. Pulsed, single-mode cavity ringdown spectroscopy. Applied Optics 1999;38(18):3951-3960. 
53 R.D. van Zee. Quantitative number-density measurements using cavity ring-down spectroscopy. CCQM Primary Methods Symposium: How Far Does the Light Shine? 4-5 April 2000, BIPM, Sèvres, France.

54 L.L.P. van Stee, U.A.T. Brinkman, H. Bagheri. Gas chromatography with atomic emission detection: a powerful technique. TRAC-Trends in Analytical Chemistry 2002;21(9-10):618626.

55 W. Jennings, E. Mittlefehldt, and P. Stremple. Analytical gas chromatography, $2^{\text {nd }}$ Edition. Academic Press, Inc. San Diego, CA USA (1997).

56 B. King and S. Westwood. GC-FID as a primary method for establishing the purity of organic CRMs used for drugs in sport analysis. Fresenius J Analytical Chemistry 2001;370:194-199.

57 K.S. Webb, D. Carter, and V.J. Barwick. International comparison (CCQM-3) on the determination of organic compounds using isotope dilution mass spectrometry: (pp 'dichlorodiphenyl) dichloroethylene (pp '-DDE) in solution. Metrologia 1999;36(2):89-99.

58 L. Mackay, Personal communication. AGAL-NARL, Sidney, Australia (2002).

59 D. Parriott, A practical Guide to HPLC Detection. Academic press, Inc. San Diego, CA USA (1992).

60 F.P. Abramson, G.E. Black, and P. Lecchi. Application of high-performance liquid chromatography with isotope-ratio mass spectrometry for measuring low levels of enrichment of underivatized materials. J Chromatographia A 2001;913(1-2):269-273.

61 B.R. Kowalski and M.B. Seasholtz. Recent developments in multivariate calibration. J Chemometrics 1991;5(3):129-145.

62 K.S. Booksh and B.R. Kowalski. Calibration method choice by comparison of model basis functions to the theoretical instrumental response function. Analytica Chimica Acta 1997;348(1-3):1-9.

63 N.M Faber, R. Bro, and P.K. Hopke. Recent developments in CANDECOMP/PARAFAC algorithms: a critical review. Chemometrics and Intelligent Laboratory Systems 2003;65(1):119-137.

64 M. Linder and R. Sundberg. Precision of prediction in second-order calibration, with focus on bilinear regression methods. J Chemometrics 2002;16(1):12-27.

65 T.S. Ma and R.C. Rittner. Modern Organic Elemental Analysis. Marcel Dekker, Inc. New York, NY USA (1979). 
66 E.F.G. Herington. Thermal methods. In: L.A.K. Stavely, ed., The characterization of chemical purity. Organic compounds, International Union of Pure and Applied Chemistry, Physical Chemistry Division, Commission on Physicochemical Measurements and Standards, Butterworth \& Co. (Publishers) Ltd, London, UK (1971), pp. 11-32.

67 T. Atake. Purity Determination Using Adiabatic Calorimeter. Poster, CCQM Primary Methods Symposium: How Far Does the Light Shine? 4-5 April 2000, BIPM, Sèvres, France.

68 H.F. Ferguson, D.J. Frurip, A.J. Pastor, L.M. Peerey, and L.F. Whiting. A review of analytical applications of calorimetry. Thermochimica Acta 2000;363(1-2):1-21.

69 T. Ozawa. Thermal analysis - review and prospect. Thermochimica Acta 2000;355(1-2):3542.

70 M.E. Brown. Determination of purity by differential scanning calorimetry (DSC). J Chemical Education 1979;56(5):310-313.

71 W.J. Mader. Phase solubility analysis. Critical Reviews in Analytical Chemistry $1970 ; 1: 193-214$.

72 W.J. Mader. Phase solubility analysis. In Organic Analysis, Volume II. J. Mitchell Jr., I.M. Kolthoff, E.S. Proskauer, and A. Weissberger, eds. Interscience Publishers, New York, NY. USA (1954), pp. 253-267.

73 G.B. Smith, G.V. Downing. Phase solubility analysis as the basis of a separation method. Analytical Chemistry 1979;51(13): 2290-2293.

74 L.T. Grady, S.E. Hays, R.H. King, H.R. Klein, W.J. Mader, D.K. Wyatt, and R.O. Zimmerer. Drug purity profiles. J Pharmaceutical Sciences. 1973;62(3):456-464.

75 J.T. Scanlon and D.E. Willis. Calculation of flame ionization detector relative response factors using the effective carbon number concept. J Chromatographic Science 1985;23(8):333-340.

76 A.R. Katritzky, E.S. Ignatchenko, R.A. Barcock, and V.S. Lobanov. Prediction of gaschromatographic retention times and response factors using a general quantitative structureproperty relationship treatment. Analytical Chemistry 1994;66(11):1799-1807.

77 M. Jalali-Heravi and Z. Garkani-Nejad. Prediction of relative response factors for flame ionization and photoionization detection using self-training artificial neural networks. J. Chromatography A 2002;950(1-2):183-194.

78 R.C. Paule and J. Mandel. Consensus values and weighting factors. J Res NBS 1982;87(5):377-385.

79 A.L. Rukhin, B.J. Biggerstaff, M.G. Vangel. Restricted maximum likelihood estimation of a common mean and the Mandel-Paule algorithm. J Stat Plan Infer 2000;83(2):319-330. 
80 M.S. Levenson, D.L. Banks, K.R. Eberhardt, L.M. Gill, W.F. Guthrie, H.K. Liu, M.G. Vangel, J.H. Yen, and N.H. Zhang. An approach to combining results from multiple methods motivated by the ISO GUM. J Res Natl Inst Stan 2000;105:571-579.

$81 \mathrm{M}$. Ballico. Calculation of key comparison reference values in the presence of non-zeromean uncertainty distributions, using the maximum-likelihood technique. Metrologia 2001;38(2):155-159.

82 L.A. Currie. Detection: International update, and some emerging di-lemmas involving calibration, the blank, and multiple detection decisions. Chemometr Intell Lab 1997;37(1):151-181. See also: C.H. Spiegelman. A discussion of issues raised by Lloyd Currie and a cross disciplinary view of detection limits and estimating parameters that are often at or near zero. Chemometr. Intell Lab 1997;37(1):183-188.

83 Analytical Methods Committee. Recommendations for the definition, estimation and use of the detection limit. Analyst 1987;112:199-204.

84 W.K. de Jongh, A last-second correction in trace analysis, International Laboratory 1986;17(Jan/Feb):62-65.

85 NIST SRM 938 4-Nitrophenol, Certificate of Analysis. National Institute of Standards and Technology, Gaithersburg, MD USA, (1995, revised from original of 1981).

86 G.N. Bowers Jr., R.B. McComb, R.G. Christensen, R. Schaffer. High-purity 4-nitrophenol: purification, characterization, and specifications for use as a spectrophotometric reference material. Clinical Chemistry 1980;26(6):724-729.

87 NIST SRM 998 Angiotensin I (Human), Certificate of Analysis. National Bureau of Standards, Washington, DC USA, (1981). See also: S.A. Margolis, R. Schaffer. NBSIR 79-1947 Development of a Standard Reference Material for Angiotensin I. National Bureau of Standards, Washington, DC USA, (1979).

88 NIST SRM 910 Sodium Pyruvate, Certificate of Analysis. National Bureau of Standards, Washington, DC USA, (1983).

89 LGC1110 p,p' - DDE 1-1-dichloro-2,2-bis(4-chlorophenyl) ethylene, Certificate, LGC (Holdings) Limited, Teddington, UK. (1997).

90 V. Barwick, S. Burke, R. Lawn, P. Roper, and R. Walker. Applications of Reference Materials in Analytical Chemisty. Royal Society of Chemistry, Cambridge UK. (2001), p 43.

91 F. Lagarde, Z. Asfari, M.J.F. Leroy, C. Demesmay, M. Ollé, A. Lamotte, P. Leperchec, E.A. Maier. Preparation of pure calibrants (arsenobetaine and arsenocholine) for arsenic speciation studies and certification of an arsenobetaine solution - CRM 626. Fresenius J Analytical Chemistry 1999;363:12-17. See also: Certified Reference Material BCR-626 Certificate of Analysis, Arsenobetaine Solution. http://irmm.jrc.cec.eu.int/rm/bcr-626.pdf 
92 J. Jacob, A.S. Lindsey, P.J. Wagstaffe. The certification of the purity of polychlorinated biphenyl isomers BCR Nos 289-298. BCR information series (CD-NA-10998-EN-C) 1987; VII:1-59. See also: Certified Reference Material,Certificate of Analysis, BCR No 289 2,4' Dichlorobiphenyl (IUPAC No. 8). http://irmm.jrc.cec.eu.int/rm/bcr-289.pdf

93 NMIJ CRM 4011-a Technical Record (3): Procedure and record of $o$-xylene production. Content of Certification. Reference Material Certificate. National Institute of Advanced Industrial Science and Technology. National Metrology Institute of Japan. 25-Mar-2002.

94 NIST SRM 911b Cholesterol, Certificate of Analysis. National Institute of Standards and Technology, Gaithersburg, MD USA, (1994, revised from original of 1988). 

. 
